\title{
Strength evaluation of intersection between stiffeners and primary supporting members considering the effect of shear force on the primary member web
}

\author{
Tetsuo Okada*, Yasumi Kawamura \\ Faculty of Engineering, Yokohama National University, Tokiwadai 79-5, Hodogaya-ku, Yokohama 240-8501 Japan \\ * Corresponding Author \\ e-mail: okada-t@ynu.ac.jp (T. Okada), kawamura-yasumi-zx@ynu.ac.jp (Y. Kawamura)
}

\begin{abstract}
The intersection between stiffeners and primary supporting members is one of the most critical details in ship hull structures. Learning from the lessons of actual damages experienced in the past, many formulae to estimate stresses around the slot cut-out structures have been developed. However, good agreement with finite element analysis or experiments has not been achieved, and the formulae have been used with empirical correction coefficient, introduced using actual damage and no-damage records or calibration based on finite element analysis results. In this study, the authors develop a consistent theoretical formula taking account of all the structural components affecting the load share of each member, in combination with the combined load effect of direct force from the longitudinal stiffener and shear force on the primary supporting member. The derived formula was verified through comparison with the results of finite element analysis and past experiments, and good accuracy was confirmed. Then, using the proposed theoretical formula, various parametric studies are carried out, and effective countermeasures to enhance the strength of slot cut-out structures are discussed. Finally, critical review on IACS CSR formulae on the slot cut-out structures is conducted. Because the rules take account of only the direct load from the longitudinal stiffeners, and do not consider the effect of the shear force on the primary supporting member, it is probable that the rules give insufficient strength when the effect of the shear force on the primary supporting member superimposes the effect of the load from the longitudinal stiffener. On the other hand, the rules may be too conservative when they cancel each other. It is shown that rational evaluation of the strength of slot cut-out structure is possible using the proposed theoretical formula.
\end{abstract}

Keyword: slot cut-out, stiffener-frame connection, classification rules, ship structural design

\section{INTRODUCTION}

Fatigue damages are the most commonly observed failures in the ship hull structures. Among thousands of structural details in the hull structures, one of the most critical details is the slot cut-out structure, where a secondary stiffener intersects with a primary supporting member, providing load path from the stiffener to the primary supporting member. The industries have experienced many kinds of fatigue damages around the slot cut-out structures since more than half a century ago.

The first massive appearance of such damages was coincident with rapid increase in the size of crude oil tankers during 1960's. Fig. 1 shows the transition of the size of the largest crude oil tankers from 1950 to 1980 (e.g. p.4 in [1]), where we can observe rapid progress of the ship size enlargement. Typical damages experienced in these days are illustrated in Fig. 2. Most commonly observed cracks initiated at the round weld toe in way of the attachment of the web stiffener to the face plate of the longitudinal stiffener, as shown as CRACK-A and CRACK-B in Fig. 2. Sometimes they were accompanied by other types of cracks CRACK-C, D, E and F in Fig. 2, mainly caused by the load redistribution due to the lost load-carrying capacity of the cracked web stiffener. Therefore, accurate assessment of the stresses at the root of the web stiffener is most important for safe design of slot cut-out structures.

Thorough investigations into the root cause of the damages were carried out then. A lot of new knowledge was obtained, and effective countermeasures were established within the design standards of each design companies ([25], pp.202-208 in [1]). Through the investigations it was revealed that among many factors, the following points were the primary factors affecting the damages:

1. In accordance with the growth in ship size, larger spacings were employed for transverse frames and longitudinal stiffeners, resulting in the increase in the load transmitted through the slot cut-out structures. 
2. To realize larger ship size efficiently, longer tanks up to about 50m length were applied. This longer tanks caused increased vertical relative deflection between the side shell structure and the longitudinal bulkhead structure, resulting in larger shear force on the bottom transverse web frame.

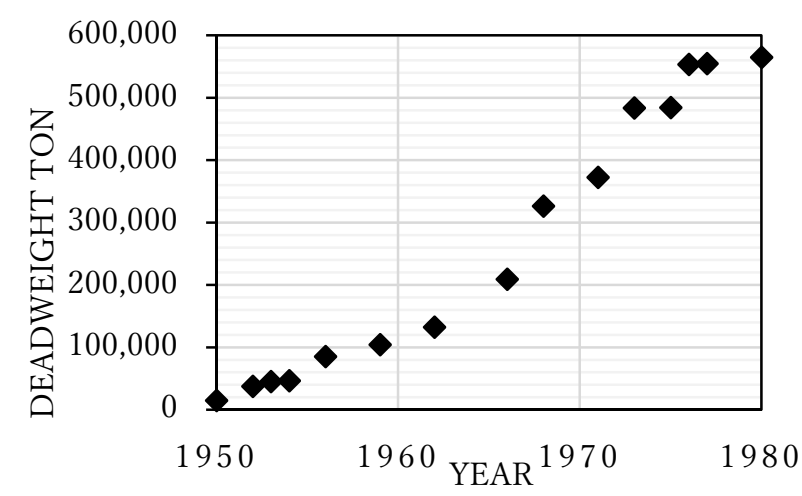

Fig. 1 Tanker size increase

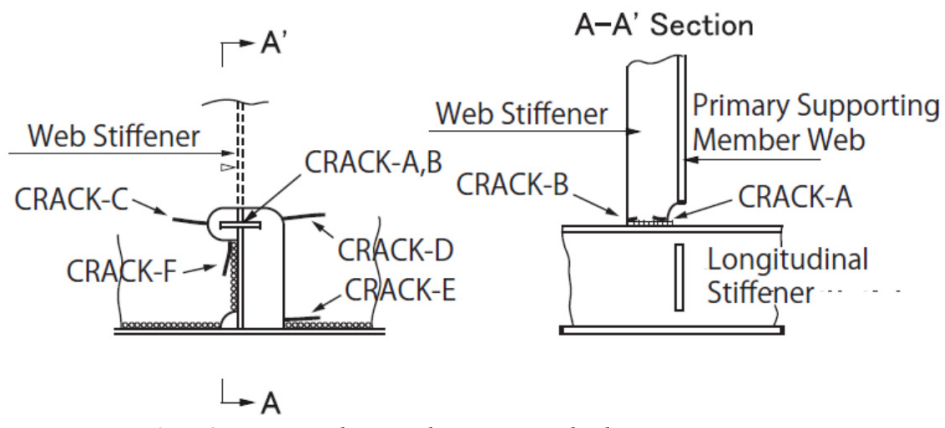

Fig. 2 Typical cracks around slot cut-out

The second point was important because the shear force on the primary supporting member induces tensile or compressive stresses in way of the connection between the web stiffener and the longitudinal stiffener, and superimpose or cancel the stresses caused by the loads transmitted from the longitudinal stiffener to the primary supporting member. The mechanism is explained in Fig. 3. The force $W$ is the load transmitted from the longitudinal stiffener to the primary supporting member, and causes tensile stress at the root of the web stiffener in this case. When the slot cut-out is open in the lower side as shown in SLOT-A in Fig. 3, the shear force $F$ in the direction as shown in SLOT-A causes compressive stress at the root of the web stiffener, and cancels the stress exerted by the force $W$. On the other hand, if the shear force $F$ is in the opposite direction as shown in SLOT-B in the figure, the stress caused by the shear force $F$ is tensile and superimpose the stress caused by the force $W$. Therefore, fatigue damages were accelerated in case of SLOT-B configuration, while the damages were suppressed in case of SLOT-A configuration.
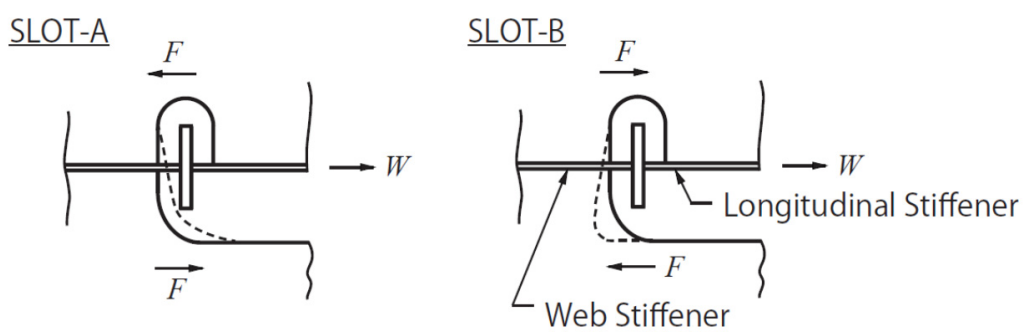

Fig. 3 Action of primary member shear force on the web stiffener stresses

Different modes of fatigue damages around the slot cut-out structures were observed later, where the crack initiated at the root of the web stiffener and propagated into the longitudinal stiffener, instead of propagating along the welding between the web stiffener and the longitudinal stiffener. The main reason of this kind of damages were the increased longitudinal stresses due to the increased application of higher tensile steels to the longitudinal stiffener [6,7]. Further, the third generation fatigue damages of slot cut-out structures were discussed by Seki et al. [8,9].

Having a look at the rules of classification societies, stress formulae and associated stress criteria in way of the stiffener-frame connection have been established in the harmonized common structural rules [10]. However, the load considered in the rules is only the load transferred from the stiffener to the primary supporting member, and the rules do not take account of the shear force on the primary supporting member. Therefore, the rules cannot explicitly incorporate the effect of the shear force on the stress at the root of the web stiffener as explained in Fig. 3. Kaase discussed enhanced strength evaluation procedure applied to the stiffener-frame connections, using fine mesh finite element analysis and extended scope of fatigue strength verification [11]. However, in this procedure, the effect of the shear force on the primary supporting member to the stress at the root of the web stiffener is not also taken into account explicitly.

In this paper, the authors firstly establish the theoretical formulation to represent the stresses at the root of the web stiffener due to the load from both the longitudinal stiffener and the shear force on the primary supporting member. Then, the results of the derived formulae are compared with the results of finite element analysis and experiments from the literature. Using the derived theoretical formula, some parametric studies are carried out with different slot cut-out structural configurations, and effective countermeasures to enhance the strength of slot cut-out structures are 
discussed. Finally, rule stipulations on slot cut-out strength are critically reviewed, and necessary improvement of the rules is proposed.

\section{DERIVATION OF THEORETICAL FORMULAE}

\subsection{Derivation of share of loads among web stiffener, primary member web and collar plate}

Learning lessons from the actual damages experienced in the past, theoretical formulation to estimate stresses around the slot cut-out structures was attempted [e.g.2,3,5,12]. Structural rules also employ closed form formulae as design criteria [10]. However, good agreement with finite element analysis or experiments has not been achieved, and the developed formulae have been used with empirical correction coefficient, introduced using actual damage and nodamage records or calibration based on finite element analysis results. The majority of the past formulae took account of three load paths between the longitudinal stiffener and the primary supporting member, i.e. the web stiffener, the primary supporting member attachment to the web of longitudinal stiffener and the collar plate. Assuming a structural configuration of the intersection of longitudinal stiffener and primary supporting member as shown in Fig. 4, the three load paths correspond to the load path $<1>$ with the sectional area of $b_{f o} \times t_{f}$, the load path $<2>$ with the sectional area of $h_{1} \times t_{w}$ and the load path $<3>$ with the sectional area of $h_{2} \times t_{c}$, respectively. However, those past formulae did not take account of the elastic spring of the primary supporting member web between the slot cut-out and the face plate of the primary supporting member (load paths $<4>$ and $<5>$ ). Therefore, the spring constant of the web stiffener was overestimated, and therefore, the share of loading born by the web stiffener was overestimated in general.

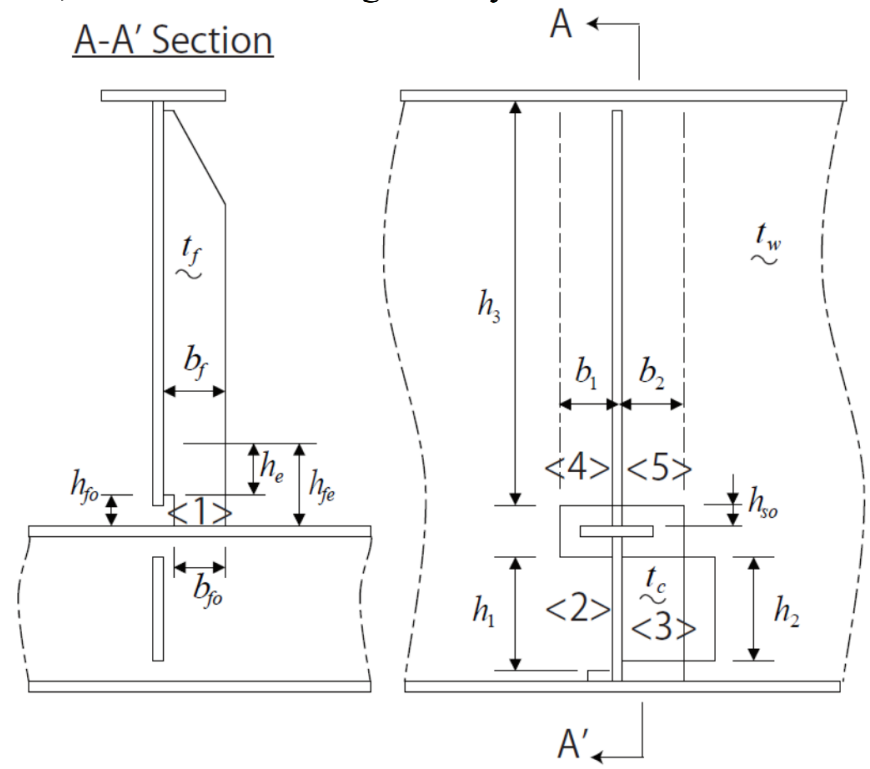

Fig. 4 Structural configuration around the intersection of longitudinal stiffener and primary supporting member

On the other hand, only the formulae introduced in Reference [3] employed exhaustive and comprehensive approach, and took account of all the structural components $<1>\sim<5>$. For the sake of the exhaustiveness, many parameters are used including less effective parameters such as the outer shell plate thickness and longitudinal spacings. Coefficients and components of the formulae were calibrated through finite element analyses of each structural component. Sometimes such coefficients must be read out from plotted graphs. In addition, the resulting share of loads among the structural components are not verified through finite element analysis nor experiments.

With regard to the loading, shear force on the primary supporting member was not necessarily taken into account in all the past formulae. Table 1 summarizes the springs and loads taken into account in the past literature. As shown in the lowest line of Table 1, in this paper, the authors develop a consistent theoretical formula, attaching importance to the following issues:

- All the effective structural components affecting the load path around the slot cut-out structures should be taken into account.

- The combined load effect of direct force from the longitudinal stiffener and shear force on the primary supporting member should also be considered.

- The derivation of the formulae should be based on elastic theories as far as possible so that the formulae have wide range of applicability. For example, pillar behavior and effective length of the web stiffener should be estimated using beam theory as explained in APPENDIX A, while it is formulated using the results of finite element analysis in Reference [3].

- The derived final formulae should be verified through finite element analyses and experiments. 
- Input parameters should be minimum and limited to effective ones and the formulae should be as simple as possible, so that the formulae can be practically applied in actual designs and rules.

Table 1 Summary of springs and loads taken into account in the past literature

\begin{tabular}{|l|c|c|c|c|c|c|c|}
\hline \multirow{2}{*}{} & \multicolumn{4}{|c|}{ Springs } & \multicolumn{2}{c|}{ Loads } \\
\cline { 2 - 8 } & $<1>$ & $<2>$ & $<3>$ & $<4>$ & $<5>$ & $\begin{array}{l}W \text { from longitudinal } \\
\text { stiffener }\end{array}$ & $\begin{array}{l}F \text { from shear force on } \\
\text { primary member }\end{array}$ \\
\hline Ochi et al. [2] & Yes & Yes & Yes & No & No & Yes & Yes \\
\hline West-Japan [3] & Yes & Yes & Yes & Yes & Yes & Yes & Yes \\
\hline SR120 [5] & Yes & Yes & Yes & No & No & Yes & Yes \\
\hline Ma et al. [12] & Yes & Yes & Yes & No & No & Yes & No \\
\hline IACS CSR [10] & Yes & Yes & Yes & No & No & Yes & No \\
\hline Present study & Yes & Yes & Yes & Yes & Yes & Yes & Yes \\
\hline
\end{tabular}

Note: "Yes" means taken into account in the formulae, "No" means not taken into account.

Fig. 5 illustrates the model of connections between each member and load transfer, under the direct force from the longitudinal, $W$, and the shear force on the primary supporting member, $F . P_{f}$ is the load transferred between the longitudinal stiffener and the web stiffener. $S_{1}$ and $S_{2}$ are the loads supported by the primary member web or collar plate at the location as shown in the figure. $k_{p}, k_{s 1}$ and $k_{s 2}$ are the spring constants corresponding to each load explained above. The load exerted on the primary member web between the slot cut-out and the face plate of the primary supporting member (the load paths $<4>$ and $<5>$ ) is not so straightforward. The load from the longitudinal stiffener flows into this primary supporting member web plate through the limited area of the web stiffener close to the longitudinal stiffener, while the shear force on the primary supporting member acts all along the length $\left(h_{3}\right)$ of the primary supporting member web. Therefore, the loads and spring constants in this area are decomposed into the component due to the load from the longitudinal stiffener $\left(S_{3 W}, S_{4 W}\right.$ and $k_{s 3 W}, k_{s 4 W}$ as shown in the figure) and the component due to the shear force on the primary supporting member $\left(S_{3 F}, S_{4 F}\right.$ and $\left.k_{s 3 F}, k_{s 4 F}\right)$.
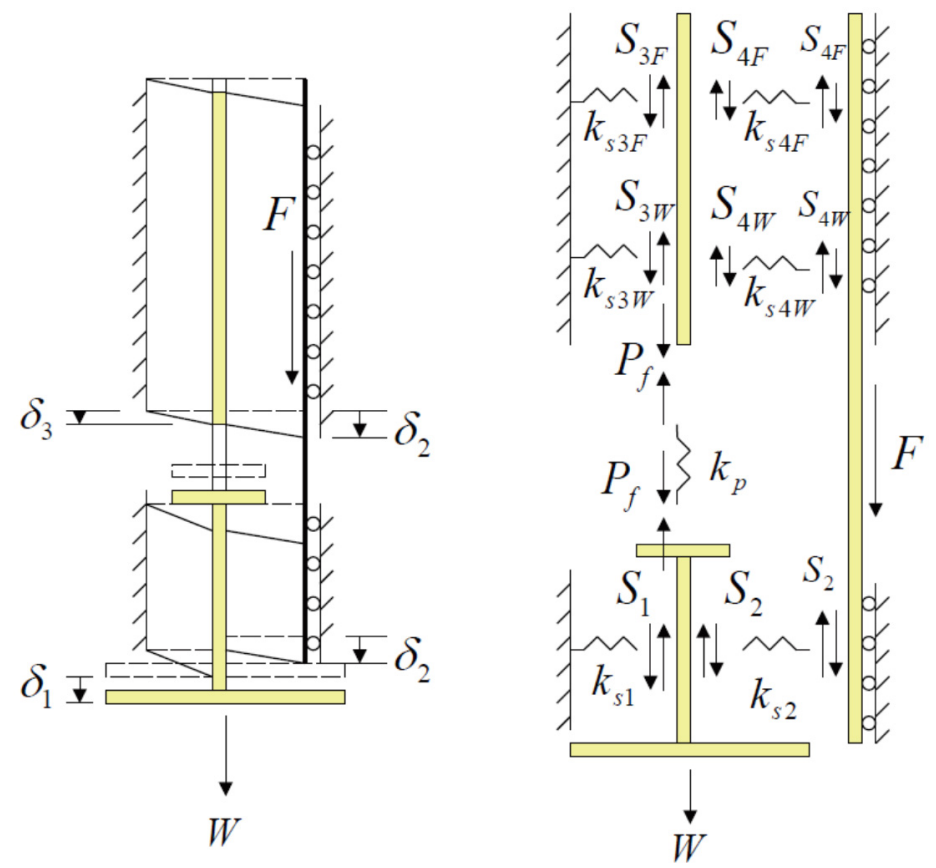

Fig. 5 Model of connections between each member and load transfer

Assuming that $\delta_{1}$ is the deflection of the lower left part of the primary supporting member web, $\delta_{3}$ is its deflection of the upper left part, and that $\delta_{2}$ is the total shear deflection due to the shear force on the primary supporting member, the load displacement relationship is expressed as Eqs.(1) (7).

$$
\begin{aligned}
& P_{f}=k_{p}\left(\delta_{1}-\delta_{3}\right) \\
& S_{1}=k_{s 1} \delta_{1}=\alpha_{1} k_{p} \delta_{1} \\
& S_{2}=k_{s 2}\left(\delta_{1}-\delta_{2}\right)=\alpha_{2} k_{p}\left(\delta_{1}-\delta_{2}\right)
\end{aligned}
$$




$$
\begin{aligned}
& S_{3 W}=k_{s 3 W} \delta_{3 W}=\alpha_{3 W} k_{p} \delta_{3 W} \\
& S_{3 F}=k_{s 3 F}\left(\delta_{3}-\delta_{3 W}\right)=\alpha_{3 F} k_{p}\left(\delta_{3}-\delta_{3 W}\right) \\
& S_{4 W}=k_{s 4 W} \delta_{3 W}=\alpha_{4 W} k_{p} \delta_{3 W} \\
& S_{4 F}=k_{s 4 F}\left(\delta_{3}-\delta_{2}-\delta_{3 W}\right)=\alpha_{4 F} k_{p}\left(\delta_{3}-\delta_{2}-\delta_{3 W}\right)
\end{aligned}
$$

where $\alpha_{1}, \alpha_{2}, \alpha_{3 W}, \alpha_{3 F}, \alpha_{4 W}$ and $\alpha_{4 F}$ are the coefficients to express each spring constant in the form of ratio to the normal spring constant of the web stiffener $k_{p} . \delta_{3 W}$ is the deflection of the primary member web due to the load from the longitudinal stiffener through the web stiffener only, and can be expressed in Eq.(8). The derivation of Eq.(8) is shown in APPENDIX B.

$$
\delta_{3 W}=\frac{1}{\left(1+\alpha_{1}+\alpha_{2}\right)\left(1+\alpha_{3 W}+\alpha_{4 W}\right)-1} \cdot \frac{W}{k_{p}}
$$

Now, $k_{p}$ can be expressed as follows using the length and the sectional area of the spring,

$$
k_{p}=\frac{E b_{f o} t_{f}}{h_{f e}}
$$

where $h_{f e}\left(=h_{f o}+h_{e}\right)$ is the equivalent length of the web stiffener, and $E$ is Young's modulus. The derivation of $h_{f e}$ is explained in APPENDIX A. Now because $k_{s 1}$ is expressed as

$$
k_{s 1}=\alpha_{1} k_{p}=\frac{G h_{1} t_{w}}{b_{1}}
$$

where $G(=E / 2(1+v))$ is the shear modulus of elasticity and $v$ is Poisson's ratio, $\alpha_{1}$ is obtained as follows.

$$
\alpha_{1}=\frac{G h_{1} t_{w}}{b_{1}} \cdot \frac{h_{f e}}{E b_{f o} t_{f}}=\frac{1}{2(1+v)} \cdot \frac{h_{1} t_{w}}{b_{f o} t_{f}} \cdot \frac{h_{f e}}{b_{1}}
$$

Similarly, $\alpha_{2}, \alpha_{3 F}$ and $\alpha_{4 F}$ can be obtained as follows.

$$
\begin{aligned}
& \alpha_{2}=\frac{1}{2(1+v)} \cdot \frac{h_{2} t_{c}}{b_{f o} t_{f}} \cdot \frac{h_{f e}}{b_{2}} \\
& \alpha_{3 F}=\frac{1}{2(1+v)} \cdot \frac{h_{3} t_{w}}{b_{f o} t_{f}} \cdot \frac{h_{f e}}{b_{1}} \\
& \alpha_{4 F}=\frac{1}{2(1+v)} \cdot \frac{h_{3} t_{w}}{b_{f o} t_{f}} \cdot \frac{h_{f e}}{b_{2}}
\end{aligned}
$$

Now, we must derive the equations for $\alpha_{3 W}$ and $\alpha_{4 W}$, which are a little more complex. First, we assume that the load transfer from the web stiffener to the primary supporting member web is limited within the zone of the equivalent length $h_{e}$ of the web stiffener, and the shear spring of the primary supporting member web is only effective within this area, and the shear deflection is further supported by normal spring from the upper part. Then, the model is illustrated in Fig. 6. The shear spring is effective within the height $h_{s}=h_{e}+h_{f o}-h_{s o}$.

Then the relation between the load $S_{3 W}$ and deflection $\delta_{3 W}$ in the upper left part can be expressed as follows, using the shear spring constant $k_{s 3 W-s}$ and the normal spring constant $k_{s 3 W-n}$. It is noted that the sectional area assumed in the calculation of the normal spring constant $k_{s 3 W-n}$ corresponds to $b_{1} / 2$, i.e., half the full sectional area, because the full width is not considered to work as a spring.

$$
\begin{aligned}
S_{3 W} & =\left(k_{s 3 W-s}+k_{s 3 W-n}\right) \delta_{3 W} \\
& =\left(\frac{G h_{s} t_{w}}{b_{1}}+\frac{E \cdot \frac{b_{1}}{2} \cdot t_{w}}{h_{3}-h_{s}}\right) \delta_{3 W} \\
& =\frac{h_{f e} t_{w}}{b_{f e} t_{f}}\left[\frac{h_{s}}{2(1+v) b_{1}}+\frac{b_{1}}{2\left(h_{3}-h_{s}\right)}\right] k_{p} \delta_{3 W}
\end{aligned}
$$




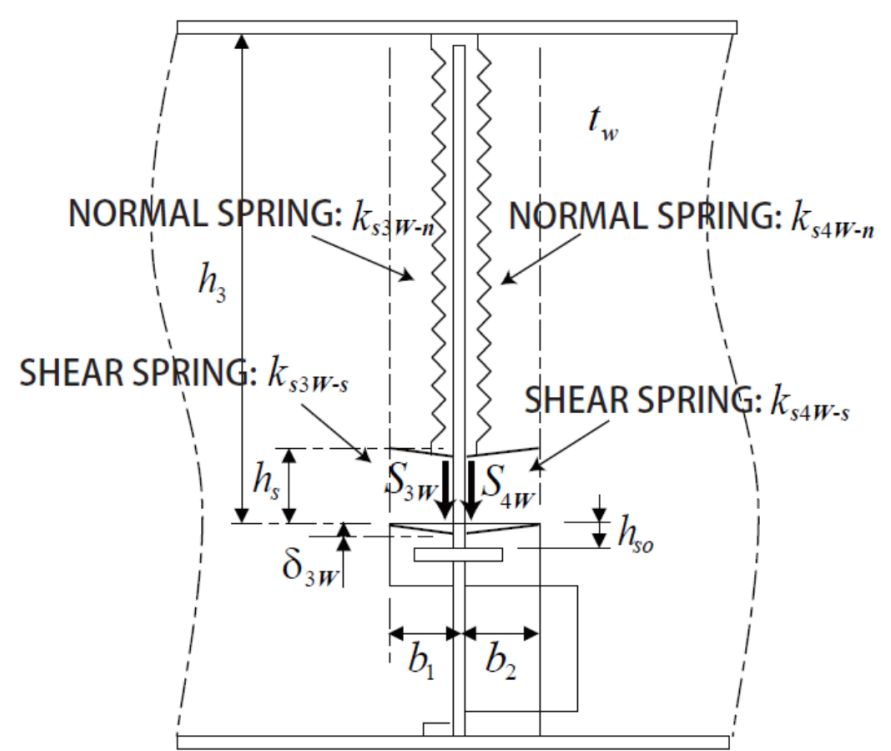

Fig. 6 Model of the spring of the upper primary supporting member web

Comparing Eq.(15) with Eq.(4), $\alpha_{3 W}$ is obtained as follows.

$$
\alpha_{3 W}=\frac{h_{f e} t_{w}}{b_{f e} t_{f}}\left[\frac{h_{s}}{2(1+v) b_{1}}+\frac{b_{1}}{2\left(h_{3}-h_{s}\right)}\right]
$$

Similarly, $\alpha_{4 W}$ is,

$$
\alpha_{4 W}=\frac{h_{f e} t_{w}}{b_{f e} t_{f}}\left[\frac{h_{s}}{2(1+v) b_{2}}+\frac{b_{2}}{2\left(h_{3}-h_{s}\right)}\right]
$$

From the equilibrium of forces at the longitudinal stiffener, the web stiffener and the primary supporting member web,

$$
\begin{aligned}
& P_{f}+S_{1}+S_{2}=W \\
& S_{3 W}+S_{3 F}+S_{4 W}+S_{4 F}=P_{f} \\
& S_{2}+S_{4 W}+S_{4 F}=-F
\end{aligned}
$$

From Eq.(18) and Eq.(19),

$$
S_{1}+S_{2}+S_{3 W}+S_{3 F}+S_{4 W}+S_{4 F}=W
$$

Subtracting Eq.(20) from Eq.(21),

$$
S_{1}+S_{3 W}+S_{3 F}=F+W
$$

From Eq.(22), we know that the shear force exerted at the left side of the slot cut-out $\left(S_{1}+S_{3 W}+S_{3 F}\right)$ is greater than the shear force at the right side of the slot cut-out $(F)$ stepwise by the amount of $W$. Therefore, the shear force increases by the amount of $n W$ at the location $n$ times longitudinal spacings away.

Substituting Eqs.(1), (2) and (3) into Eq.(18),

$$
k_{p}\left(\delta_{1}-\delta_{3}\right)+\alpha_{1} k_{p} \delta_{1}+\alpha_{2} k_{p}\left(\delta_{1}-\delta_{2}\right)=W \quad \text { or } \quad\left(1+\alpha_{1}+\alpha_{2}\right) \delta_{1}-\alpha_{2} \delta_{2}-\delta_{3}=\frac{W}{k_{p}}
$$

Substituting Eqs.(3), (6) and (7) into Eq.(20),

$$
\begin{aligned}
& \alpha_{2} k_{p}\left(\delta_{1}-\delta_{2}\right)+\alpha_{4 W} k_{p} \delta_{3 W}+\alpha_{4 F} k_{p}\left(\delta_{3}-\delta_{2}-\delta_{3 W}\right)=-F \quad \text { or } \\
& -\alpha_{2} \delta_{1}+\left(\alpha_{2}+\alpha_{4 F}\right) \delta_{2}-\alpha_{4 F} \delta_{3}=\frac{F}{k_{p}}+\left(\alpha_{4 W}-\alpha_{4 F}\right) \delta_{3 W}
\end{aligned}
$$

Putting Eqs.(1), (4), (5), (6) and (7) into Eq.(19),

$$
\begin{aligned}
& \alpha_{3 W} k_{p} \delta_{3 W}+\alpha_{3 F} k_{p}\left(\delta_{3}-\delta_{3 W}\right)+\alpha_{4 W} k_{p} \delta_{3 W}+\alpha_{4 F} k_{p}\left(\delta_{3}-\delta_{2}-\delta_{3 W}\right)=k_{p}\left(\delta_{1}-\delta_{3}\right) \quad \text { or } \\
& -\delta_{1}-\alpha_{4 F} \delta_{2}+\left(1+\alpha_{3 F}+\alpha_{4 F}\right) \delta_{3}=\left(-\alpha_{3 W}+\alpha_{3 F}-\alpha_{4 W}+\alpha_{4 F}\right) \delta_{3 W}
\end{aligned}
$$

In a matrix form, Eqs.(23) (25) are rewritten as follows. 


$$
\left(\begin{array}{ccc}
\left(1+\alpha_{1}+\alpha_{2}\right) & -\alpha_{2} & -1 \\
-\alpha_{2} & \left(\alpha_{2}+\alpha_{4 F}\right) & -\alpha_{4 F} \\
-1 & -\alpha_{4 F} & \left(1+\alpha_{3 F}+\alpha_{4 F}\right)
\end{array}\right)\left(\begin{array}{l}
\delta_{1} \\
\delta_{2} \\
\delta_{3}
\end{array}\right)=\left(\begin{array}{c}
\frac{W}{k_{p}} \\
\frac{F}{k_{p}}+\left(\alpha_{4 W}-\alpha_{4 F}\right) \delta_{3 W} \\
\left(-\alpha_{3 W}+\alpha_{3 F}-\alpha_{4 W}+\alpha_{4 F}\right) \delta_{3 W}
\end{array}\right)=\left(\begin{array}{c}
\frac{W}{k_{p}} \\
\left(n-\frac{1}{2}\right) \frac{W}{k_{p}}+\left(\alpha_{4 W}-\alpha_{4 F}\right) \delta_{3 W} \\
\left(-\alpha_{3 W}+\alpha_{3 F}-\alpha_{4 W}+\alpha_{4 F}\right) \delta_{3 W}
\end{array}\right)
$$

where

$$
F=\left(n-\frac{1}{2}\right) W,
$$

and $n$ is the number of longitudinal stiffeners intersecting the primary supporting member, between the subject location and the section where the shear force is zero. Further, putting Eq.(8) into Eq.(26), the equation reduces to,

$$
\left(\begin{array}{ccc}
\left(1+\alpha_{1}+\alpha_{2}\right) & -\alpha_{2} & -1 \\
-\alpha_{2} & \left(\alpha_{2}+\alpha_{4 F}\right) & -\alpha_{4 F} \\
-1 & -\alpha_{4 F} & \left(1+\alpha_{3 F}+\alpha_{4 F}\right)
\end{array}\right)\left(\begin{array}{c}
\delta_{1} \\
\delta_{2} \\
\delta_{3}
\end{array}\right)=\left(\begin{array}{c}
1 \\
n-\frac{1}{2}+\frac{\alpha_{4 W}-\alpha_{4 F}}{\left(1+\alpha_{1}+\alpha_{2}\right)\left(1+\alpha_{3 W}+\alpha_{4 W}\right)-1} \\
\frac{-\alpha_{3 W}+\alpha_{3 F}-\alpha_{4 W}+\alpha_{4 F}}{\left(1+\alpha_{1}+\alpha_{2}\right)\left(1+\alpha_{3 W}+\alpha_{4 W}\right)-1}
\end{array}\right) \frac{W}{k_{p}}
$$

Solving Eq.(28), the deflections are determined and expressed as follows.

$$
\begin{aligned}
& \left(\begin{array}{l}
\delta_{1} \\
\delta_{2} \\
\delta_{3}
\end{array}\right)=\left(\begin{array}{ccc}
\left(1+\alpha_{1}+\alpha_{2}\right) & -\alpha_{2} & -1 \\
-\alpha_{2} & \left(\alpha_{2}+\alpha_{4 F}\right) & -\alpha_{4 F} \\
-1 & -\alpha_{4 F} & \left(1+\alpha_{3 F}+\alpha_{4 F}\right)
\end{array}\right)^{-1}\left(\begin{array}{c}
1 \\
n-\frac{1}{2}+\frac{\alpha_{4 W}-\alpha_{4 F}}{\left(1+\alpha_{1}+\alpha_{2}\right)\left(1+\alpha_{3 W}+\alpha_{4 W}\right)-1} \\
\frac{-\alpha_{3 W}+\alpha_{3 F}-\alpha_{4 W}+\alpha_{4 F}}{\left(1+\alpha_{1}+\alpha_{2}\right)\left(1+\alpha_{3 W}+\alpha_{4 W}\right)-1}
\end{array}\right) \frac{W}{k_{p}} \\
& =\frac{1}{|A|}\left(\begin{array}{ccc}
\left(\alpha_{2}+\alpha_{4 F}\right)\left(1+\alpha_{3 F}+\alpha_{4 F}\right)-\alpha_{4 F}{ }^{2} & \alpha_{2}\left(1+\alpha_{3 F}+\alpha_{4 F}\right)+\alpha_{4 F} & \alpha_{2} \alpha_{4 F}+\alpha_{2}+\alpha_{4 F} \\
\alpha_{2}\left(1+\alpha_{3 F}+\alpha_{4 F}\right)+\alpha_{4 F} & \left(1+\alpha_{1}+\alpha_{2}\right)\left(1+\alpha_{3 F}+\alpha_{4 F}\right)-1 & \alpha_{4 F}\left(1+\alpha_{1}+\alpha_{2}\right)+\alpha_{2} \\
\alpha_{2} \alpha_{4 F}+\alpha_{2}+\alpha_{4 F} & \alpha_{4 F}\left(1+\alpha_{1}+\alpha_{2}\right)+\alpha_{2} & \left(1+\alpha_{1}+\alpha_{2}\right)\left(\alpha_{2}+\alpha_{4 F}\right)-\alpha_{2}{ }^{2}
\end{array}\right) . \\
& \left(\begin{array}{c}
n-\frac{1}{2}+\frac{\alpha_{4 W}-\alpha_{4 F}}{\left(1+\alpha_{1}+\alpha_{2}\right)\left(1+\alpha_{3 W}+\alpha_{4 W}\right)-1} \\
\frac{-\alpha_{3 W}+\alpha_{3 F}-\alpha_{4 W}+\alpha_{4 F}}{\left(1+\alpha_{1}+\alpha_{2}\right)\left(1+\alpha_{3 W}+\alpha_{4 W}\right)-1}
\end{array}\right) \frac{W}{k_{p}}
\end{aligned}
$$

where

$$
|A|=\left(1+\alpha_{1}+\alpha_{2}\right)\left(\alpha_{2}+\alpha_{4 F}\right)\left(1+\alpha_{3 F}+\alpha_{4 F}\right)-2 \alpha_{2} \alpha_{4 F}-\alpha_{2}-\alpha_{4 F}-\alpha_{2}^{2}\left(1+\alpha_{3 F}+\alpha_{4 F}\right)-\alpha_{4 F}{ }^{2}\left(1+\alpha_{1}+\alpha_{2}\right)
$$

When we rewrite Eq.(29) as follows, introducing $\beta_{1}, \beta_{2}$ and $\beta_{3}$,

$$
\left(\begin{array}{l}
\delta_{1} \\
\delta_{2} \\
\delta_{3}
\end{array}\right)=\left(\begin{array}{l}
\beta_{1} \\
\beta_{2} \\
\beta_{3}
\end{array}\right) \frac{W}{k_{p}}
$$

then, putting back the obtained deflections to Eqs.(1) (3), share of loads among the web stiffener, the primary supporting member web and the collar plate is finally obtained as follows.

$$
\begin{aligned}
& P_{f}=\left(\beta_{1}-\beta_{3}\right) W \\
& S_{1}=\alpha_{1} \beta_{1} W \\
& S_{2}=\alpha_{2}\left(\beta_{1}-\beta_{2}\right) W
\end{aligned}
$$




\subsection{Stress distribution along the root of web stiffener due to the bending of web stiffener}

Web stiffeners are usually attached on one side of the primary supporting member web. Because of this eccentricity, the load from the longitudinal stiffener causes bending of the web stiffener, and the stress at the corner of the scallop becomes much larger than the average stress along the root of the web stiffener as shown in Fig. 7. To derive the stress distribution theoretically, the web stiffener is modeled as shown in Fig.8, supported by the primary supporting member through shear springs at the connection between the web stiffener and the primary supporting member web. The primary supporting member is assumed to be quite rigid in this case.

From the equilibrium of forces and moments of a small part of the web stiffener as shown in Fig. 8,

$$
\begin{aligned}
& \frac{d F_{f}}{d x}=0 \\
& \frac{d P}{d x}=f_{w} \\
& \frac{d M_{f}}{d x}-F_{f}-\frac{b_{f}}{2} f_{w}=0
\end{aligned}
$$

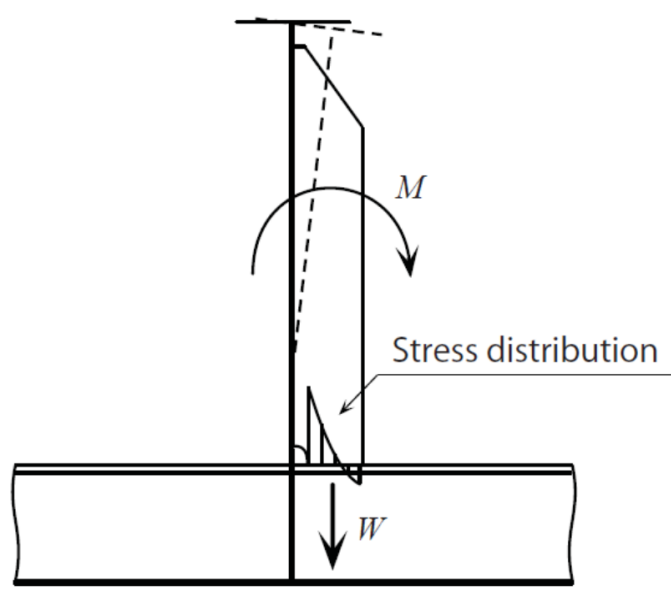

Fig. 7 bending of web stiffener

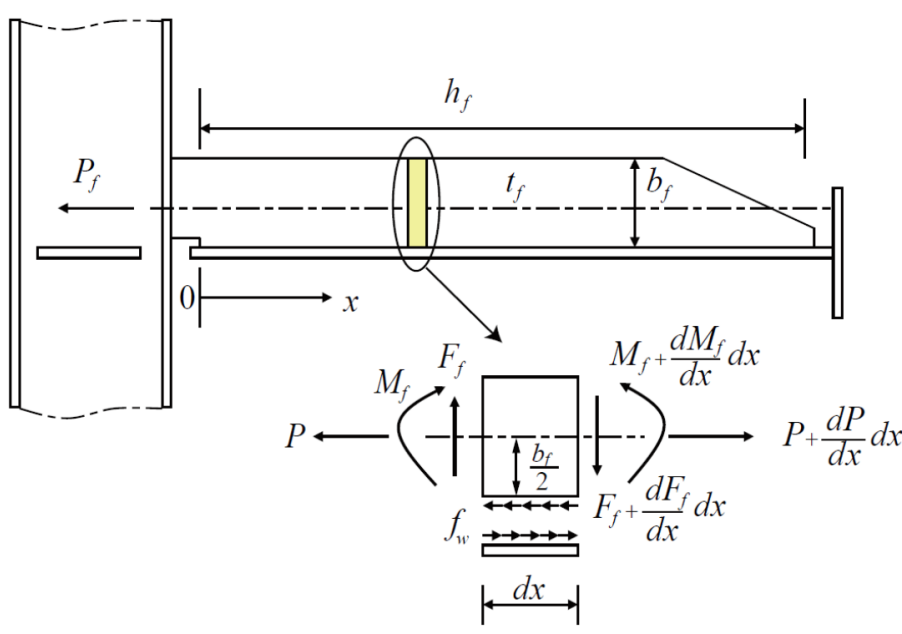

Fig. 8 Mathematical representation of web stiffener

In addition, axial force $P$ and bending moment $M_{f}$ of the web stiffener are expressed as:

$$
\begin{aligned}
& P=E A_{f} \frac{d u_{f}}{d x} \\
& M_{f}=-E I_{f} \frac{d^{2} y_{f}}{d x^{2}}
\end{aligned}
$$

where $u_{f}$ is the elongation of the web stiffener, $y_{f}$ is the deflection of the web stiffener, $A_{f}$ is the sectional area of the web stiffener $\left(=b_{f} t_{f}\right)$, and $I_{f}$ is the moment of inertia of section of the web stiffener $\left(=b_{f}^{3} t_{f} / 12\right)$.

At the connection of the stiffener to the web of the primary supporting member,

$$
f_{w}=E k_{w} u_{w}=E k_{w}\left(u_{f}-\frac{b_{f}}{2} \frac{d y_{f}}{d x}\right)
$$

where $k_{w}$ is the non-dimensional constant of the shear spring at the connection.

Now that we have six equations (35) (40) for six unknown parameters, $P, F_{f}, M_{f}, f_{w}, u_{f}$ and $y_{f}$, we can solve these equations under relevant boundary conditions.

Differentiating Eq.(37) with $x$,

$$
\frac{d^{2} M_{f}}{d x^{2}}-\frac{d F_{f}}{d x}-\frac{b_{f}}{2} \frac{d f_{w}}{d x}=0
$$

Putting Eqs.(35) and (36) into Eq.(41),

$$
\frac{d^{2} M_{f}}{d x^{2}}=\frac{b_{f}}{2} \frac{d^{2} P}{d x^{2}}
$$

Differentiating Eq.(40) with $x$, 


$$
\frac{1}{E k_{w}} \frac{d f_{w}}{d x}=\frac{d u_{f}}{d x}-\frac{b_{f}}{2} \frac{d^{2} y_{f}}{d x^{2}}
$$

Putting Eqs.(36) (38) and (39) into Eq.(43),

$$
\frac{1}{k_{w}} \frac{d^{2} P}{d x^{2}}=\frac{P}{A_{f}}+\frac{b_{f}}{2 I_{f}} M_{f}
$$

Differentiating Eq.(44) with $x$ twice,

$$
\frac{1}{k_{w}} \frac{d^{4} P}{d x^{4}}=\frac{1}{A_{f}} \frac{d^{2} P}{d x^{2}}+\frac{b_{f}}{2 I_{f}} \frac{d^{2} M_{f}}{d x^{2}}
$$

Putting Eq.(42) into Eq.(45),

$$
\frac{d^{4} P}{d x^{4}}-k_{w}\left(\frac{1}{A_{f}}+\frac{b_{f}^{2}}{4 I_{f}}\right) \frac{d^{2} P}{d x^{2}}=0
$$

Further, introducing non-dimensional parameter $\xi=x / h_{f}$,

$$
\frac{d^{4} P}{d \xi^{4}}-\lambda^{2} \frac{d^{2} P}{d \xi^{2}}=0
$$

where

$$
\begin{aligned}
& \lambda^{2}=k_{w} h_{f}{ }^{2}\left(\frac{1}{A_{f}}+\frac{b_{f}{ }^{2}}{4 I_{f}}\right)=k_{w} \frac{h_{f}{ }^{2}}{A_{f}} \frac{1}{I_{f}}\left(I_{f}+A_{f} \frac{b_{f}^{2}}{4}\right)=k_{w} \frac{h_{f}{ }^{2}}{A_{f}} \frac{I_{o}}{I_{f}}=k_{w} \frac{h_{f}{ }^{2}}{b_{f} t_{f}} \frac{\frac{1}{3} b_{f}{ }^{3} t_{f}}{\frac{1}{12} b_{f}{ }^{3} t_{f}}=4 k_{w} \frac{h_{f}{ }^{2}}{b_{f} t_{f}}=4 k_{w}\left(\frac{h_{f}}{b_{f}}\right)^{2} \frac{b_{f}}{t_{f}} \\
& \lambda=2 \sqrt{k_{w}\left(\frac{b_{f}}{t_{f}}\right)}\left(\frac{h_{f}}{b_{f}}\right) \\
& I_{o}=I_{f}+A_{f} \frac{b_{f}{ }^{2}}{4}=\frac{1}{12} b_{f}{ }^{3} t_{f}+\frac{1}{4} b_{f}{ }^{3} t_{f}=\frac{1}{3} b_{f}{ }^{3} t_{f}
\end{aligned}
$$

Eq.(47) is the fundamental differential equation in this case, whose solution is expressed as:

$$
P=C_{1}+C_{2} \xi+C_{3} \cosh \lambda \xi+C_{4} \sinh \lambda \xi
$$

Putting Eq.(51) into Eq.(44), the bending moment exerted on the web stiffener is:

$$
\begin{aligned}
M_{f} & =\frac{2 I_{f}}{b_{f}}\left(\frac{1}{k_{w}} \frac{d^{2} P}{d x^{2}}-\frac{P}{A_{f}}\right)=\frac{2 I_{f}}{b_{f}}\left(\frac{1}{k_{w} h_{f}{ }^{2}} \frac{d^{2} P}{d \xi^{2}}-\frac{P}{A_{f}}\right) \\
& =\frac{2 I_{f}}{b_{f}}\left\{\frac{1}{k_{w} h_{f}{ }^{2}}\left(C_{3} \lambda^{2} \cosh \lambda \xi+C_{4} \lambda^{2} \sinh \lambda \xi\right)-\frac{1}{A_{f}}\left(C_{1}+C_{2} \xi+C_{3} \cosh \lambda \xi+C_{4} \sinh \lambda \xi\right)\right\} \\
& =\frac{2 I_{f}}{b_{f}}\left\{\left(\frac{\lambda^{2}}{k_{w} h_{f}{ }^{2}}-\frac{1}{A_{f}}\right)\left(C_{3} \cosh \lambda \xi+C_{4} \sinh \lambda \xi\right)-\frac{1}{A_{f}}\left(C_{1}+C_{2} \xi\right)\right\} \\
& =\frac{2 I_{f}}{b_{f}}\left\{\frac{b_{f}{ }^{2}}{4 I_{f}}\left(C_{3} \cosh \lambda \xi+C_{4} \sinh \lambda \xi\right)-\frac{1}{A_{f}}\left(C_{1}+C_{2} \xi\right)\right\}=\frac{b_{f}}{2}\left(C_{3} \cosh \lambda \xi+C_{4} \sinh \lambda \xi\right)-\frac{b_{f}}{6}\left(C_{1}+C_{2} \xi\right) \\
& =\frac{b_{f}}{2}\left\{\left(C_{3} \cosh \lambda \xi+C_{4} \sinh \lambda \xi\right)-\frac{1}{3}\left(C_{1}+C_{2} \xi\right)\right\}
\end{aligned}
$$

where the following relationship is used.

$$
\frac{\lambda^{2}}{k_{w} h_{f}{ }^{2}}-\frac{1}{A_{f}}=\frac{1}{A_{f}} \frac{I_{o}}{I_{f}}-\frac{1}{A_{f}}=\frac{1}{A_{f} I_{f}}\left(I_{o}-I_{f}\right)=\frac{1}{A_{f} I_{f}} A_{f} \frac{b_{f}{ }^{2}}{4}=\frac{b_{f}{ }^{2}}{4 I_{f}}
$$

From Eq.(36), the shear force working at the connection is:

$$
f_{w}=\frac{d P}{d x}=\frac{1}{h_{f}} \frac{d P}{d \xi}=\frac{1}{h_{f}}\left\{C_{2}+\lambda\left(C_{3} \sinh \lambda \xi+C_{4} \cosh \lambda \xi\right)\right\}
$$


Putting Eqs.(52) and (54) into Eq.(37), the shear force in the web stiffener is:

$$
\begin{aligned}
F_{f} & =\frac{d M_{f}}{d x}-\frac{b_{f}}{2} f_{w}=\frac{1}{h_{f}} \frac{d M_{f}}{d \xi}-\frac{b_{f}}{2} f_{w} \\
& =\frac{b_{f}}{2 h_{f}}\left\{\lambda\left(C_{3} \sinh \lambda \xi+C_{4} \cosh \lambda \xi\right)-\frac{1}{3} C_{2}\right\}-\frac{b_{f}}{2 h_{f}}\left\{C_{2}+\lambda\left(C_{3} \sinh \lambda \xi+C_{4} \cosh \lambda \xi\right)\right\} \\
& =\frac{b_{f}}{2 h_{f}}\left(-\frac{4}{3}\right) C_{2}=-\frac{2}{3} \frac{b_{f}}{h_{f}} C_{2}
\end{aligned}
$$

The constants, $C_{1}, C_{2}, C_{3}$ and $C_{4}$ are determined under relevant boundary conditions. Considering the boundary conditions as shown in Eq.(56), the constants are finally obtained as shown in Eq.(57).

$$
\begin{aligned}
& \begin{cases}F_{f}=0 & \text { along the web stiffener } \\
P=P_{f} & \text { at } \xi=0 \\
P=0 & \text { at } \quad \xi=1 \\
M_{f}=0 & \text { at } \quad \xi=1\end{cases} \\
& C_{1}=0, \quad C_{2}=0, \quad C_{3}=P_{f}, \quad C_{4}=-P_{f} \frac{1}{\tanh \lambda}
\end{aligned}
$$

Putting the obtained constants into Eqs.(51) (52) and (54), the axial force and the bending moment in the web stiffener and the shear force at the connection are obtained as follows:

$$
\begin{aligned}
& P=C_{3} \cosh \lambda \xi+C_{4} \sinh \lambda \xi=P_{f}\left(\cosh \lambda \xi-\frac{\sinh \lambda \xi}{\tanh \lambda}\right) \\
& M_{f}=\frac{b_{f}}{2}\left(C_{3} \cosh \lambda \xi+C_{4} \sinh \lambda \xi\right)=P_{f} \frac{b_{f}}{2}\left(\cosh \lambda \xi-\frac{\sinh \lambda \xi}{\tanh \lambda}\right) \\
& f_{w}=\frac{\lambda}{h_{f}}\left(C_{3} \sinh \lambda \xi+C_{4} \cosh \lambda \xi\right)=P_{f} \frac{\lambda}{h_{f}}\left(\sinh \lambda \xi-\frac{\cosh \lambda \xi}{\tanh \lambda}\right)
\end{aligned}
$$

Then, putting the obtained $M_{f}$ of Eq.(59) into Eq.(39),

$$
\begin{aligned}
& -E I_{f} \frac{d^{2} y_{f}}{d x^{2}}=P_{f} \frac{b_{f}}{2}\left(\cosh \lambda \xi-\frac{\sinh \lambda \xi}{\tanh \lambda}\right) \\
& \therefore \frac{d^{2} y_{f}}{d \xi^{2}}=-\frac{P_{f} b_{f} h_{f}^{2}}{2 E I_{f}}\left(\cosh \lambda \xi-\frac{\sinh \lambda \xi}{\tanh \lambda}\right) \\
& \frac{d y_{f}}{d \xi}=-\frac{P_{f} b_{f} h_{f}^{2}}{2 E I_{f}}\left(\frac{1}{\lambda} \sinh \lambda \xi-\frac{\cosh \lambda \xi}{\lambda \tanh \lambda}\right)+C_{5}
\end{aligned}
$$

Considering the boundary condition of

$$
\frac{d y_{f}}{d \xi}=0 \quad \text { at } \xi=0
$$

The integral constant $C_{5}$ is obtained as:

$$
\begin{aligned}
& -\frac{P_{f} b_{f} h_{f}{ }^{2}}{2 E I_{f}} \cdot\left(-\frac{1}{\lambda \tanh \lambda}\right)+C_{5}=0 \\
& \therefore C_{5}=-\frac{P_{f} b_{f} h_{f}{ }^{2}}{2 E I_{f}} \cdot \frac{1}{\lambda \tanh \lambda} \\
& \therefore \frac{d y_{f}}{d \xi}=-\frac{P_{f} b_{f} h_{f}{ }^{2}}{2 E I_{f}}\left(\frac{1}{\lambda} \sinh \lambda \xi-\frac{\cosh \lambda \xi-1}{\lambda \tanh \lambda}\right)
\end{aligned}
$$

Next, substituting Eq.(58) into Eq.(38), 


$$
\begin{aligned}
& P=E A_{f} \frac{d u_{f}}{d x}=P_{f}\left(\cosh \lambda \xi-\frac{\sinh \lambda \xi}{\tanh \lambda}\right) \\
& \frac{d u_{f}}{d \xi}=\frac{P_{f} h_{f}}{E A_{f}}\left(\cosh \lambda \xi-\frac{\sinh \lambda \xi}{\tanh \lambda}\right) \\
& u_{f}=\frac{P_{f} h_{f}}{E A_{f}}\left(\frac{1}{\lambda} \sinh \lambda \xi-\frac{\cosh \lambda \xi}{\lambda \tanh \lambda}\right)+C_{6}
\end{aligned}
$$

Considering the boundary condition of

$$
u_{f}=0 \quad \text { at } \xi=0
$$

The integral constant $C_{6}$ is obtained as:

$$
\begin{aligned}
& \frac{P_{f} h_{f}}{E A_{f}}\left(-\frac{1}{\lambda \tanh \lambda}\right)+C_{6}=0 \\
& C_{6}=\frac{P_{f} h_{f}}{E A_{f}} \cdot \frac{1}{\lambda \tanh \lambda} \\
& \therefore u_{f}=\frac{P_{f} h_{f}}{E A_{f}}\left(\frac{1}{\lambda} \sinh \lambda \xi-\frac{\cosh \lambda \xi-1}{\lambda \tanh \lambda}\right)
\end{aligned}
$$

Then, the axial stress in way of the center of the web stiffener is obtained as follows.

$$
\sigma_{f}=E \frac{d u_{f}}{d x}=\frac{E}{h_{f}} \cdot \frac{d u_{f}}{d \xi}=\frac{P_{f}}{A_{f}}\left(\cosh \lambda \xi-\frac{\sinh \lambda \xi}{\tanh \lambda}\right)
$$

We can confirm from this equation that when $x=(\xi=) 0, \sigma_{f}$ agrees with the nominal stress, i.e., axial force at the root, $P_{f}$, divided by the sectional area $A_{f}$.

Next, as shown in Eq.(40), the axial displacement in way of the connection between the web stiffener and the primary supporting member web, $u_{w}$, is expressed as follows,

$$
u_{w}=u_{f}-\frac{b_{f}}{2} \frac{d y_{f}}{d x}=u_{f}-\frac{b_{f}}{2 h_{f}} \frac{d y_{f}}{d \xi}
$$

Putting Eq.(66) into Eq.(74),

$$
u_{w}=u_{f}+\frac{b_{f}}{2 h_{f}} \cdot \frac{P_{f} b_{f} h_{f}{ }^{2}}{2 E I_{f}}\left(\frac{1}{\lambda} \sinh \lambda \xi-\frac{\cosh \lambda \xi-1}{\lambda \tanh \lambda}\right)=u_{f}+\frac{P_{f} b_{f}{ }^{2} h_{f}}{4 E I_{f}}\left(\frac{1}{\lambda} \sinh \lambda \xi-\frac{\cosh \lambda \xi-1}{\lambda \tanh \lambda}\right)
$$

Thus, the axial stress in way is:

$$
\begin{aligned}
\sigma_{w} & =E \frac{d u_{w}}{d x}=\frac{E}{h_{f}} \cdot \frac{d u_{w}}{d \xi}=\frac{E}{h_{f}} \cdot \frac{d u_{f}}{d \xi}+\frac{E}{h_{f}} \cdot \frac{P_{f} b_{f}{ }^{2} h_{f}}{4 E I_{f}}\left(\cosh \lambda \xi-\frac{\sinh \lambda \xi}{\tanh \lambda}\right) \\
& =\sigma_{f}+\frac{P_{f} b_{f}^{2}}{4 I_{f}}\left(\cosh \lambda \xi-\frac{\sinh \lambda \xi}{\tanh \lambda}\right)
\end{aligned}
$$

Finally the stress at the scallop, where $x=(\xi=) 0$, is obtained as follows:

$$
\left.\sigma_{w}\right|_{x=0}=\left.\sigma_{f}\right|_{x=0}+\frac{P_{f} b_{f}^{2}}{4 I_{f}}=\frac{P_{f}}{A_{f}}\left(1+\frac{b_{f}^{2} A_{f}}{4 I_{f}}\right)
$$

Now that

$$
A_{f}=b_{f} t_{f} \quad I_{f}=\frac{1}{12} b_{f}^{3} t_{f}
$$

Then,

$$
\left.\sigma_{w}\right|_{x=0}=\frac{P_{f}}{A_{f}}\left(1+\frac{12 b_{f}{ }^{2} b_{f} t_{f}}{4 b_{f}{ }^{3} t_{f}}\right)=4 \cdot \frac{P_{f}}{A_{f}}
$$

We can observe that the maximum stress is exerted in way of the connection between the web stiffener and the primary supporting member web, and its magnitude is 4 times the nominal (average) axial stress $\sigma_{f}$ as shown in Fig. 
9 at $x=0$, irrespective of the scantlings and structural configurations. This may be valid when the primary supporting member web can be considered to be sufficiently rigid.

Next, let us consider the effect of the scallop at the root of the web stiffener. Existence of the scallop causes eccentricity as shown in Fig. 10.

Considering the equilibrium of the moment of the left part of Fig. 10,

$$
M_{o}=\left.M_{f}\right|_{x=0}+P_{f} \cdot \frac{b_{f}-b_{f o}}{2}
$$

From Eq.(59), we know that

$$
\left.M_{f}\right|_{x=0}=\frac{P_{f} b_{f}}{2}
$$
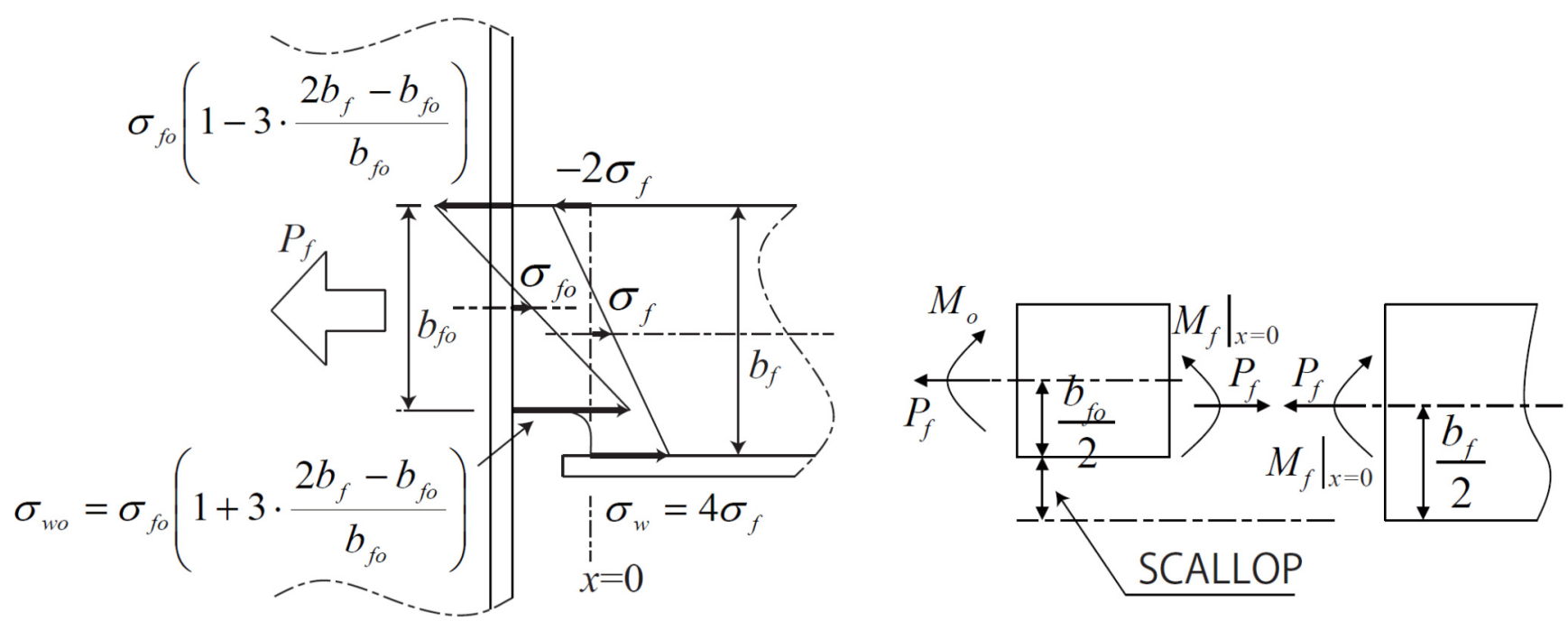

Fig. 9 Stress distribution along the root of web stiffener

Fig. 10 Model to account for the effect of the scallop

Therefore,

$$
M_{o}=\frac{P_{f} b_{f}}{2}+P_{f} \cdot \frac{b_{f}-b_{f o}}{2}=\frac{P_{f}\left(2 b_{f}-b_{f o}\right)}{2}
$$

Then, the stress, $\sigma_{w o}$, at the corner of the scallop in way of the connection between the web stiffener and the longitudinal stiffener is obtained as follows, as the sum of the average axial stress in the first term $\left(\sigma_{f o}\right)$ and the bending stress in the second term.

$$
\sigma_{w o}=\frac{P_{f}}{t_{f} b_{f o}}+\frac{M_{o}}{Z_{o}}=\sigma_{f o}+\frac{P_{f}\left(2 b_{f}-b_{f o}\right)}{2} \cdot \frac{6}{t_{f} b_{f o}{ }^{2}}=\sigma_{f o}\left(1+3 \cdot \frac{2 b_{f}-b_{f o}}{b_{f o}}\right)
$$

where $Z_{o}$ is the section modulus of the section in way of the connection of the web stiffener to the longitudinal stiffener. This stress distribution is also shown in Fig. 9 along the connection between the web stiffener and the longitudinal stiffener.

\section{VERIFICATION THROUGH FINITE ELEMENT ANALYSIS AND EXPERIMENTS}

\subsection{Middle scale experiment}

Ochi et al.[2] carried out a middle scale experiment to reveal the stress distribution around the slot cut-out structures. They employed a one-third scale model of a side transverse web of an oil tanker, whose arrangement is shown in Fig. 11. Five longitudinal stiffeners were arranged intersecting with the primary supporting member, which is simply supported at both the ends. Total loading of 10 tons were applied, equally divided to each longitudinal stiffener. Therefore, the loading transferred at each intersection was 2 tons, and maximum shear force of 5 tons were exerted at both the ends of the primary supporting member. Strain gauges were arranged along the root of the web stiffener and around the slot cut-out. 


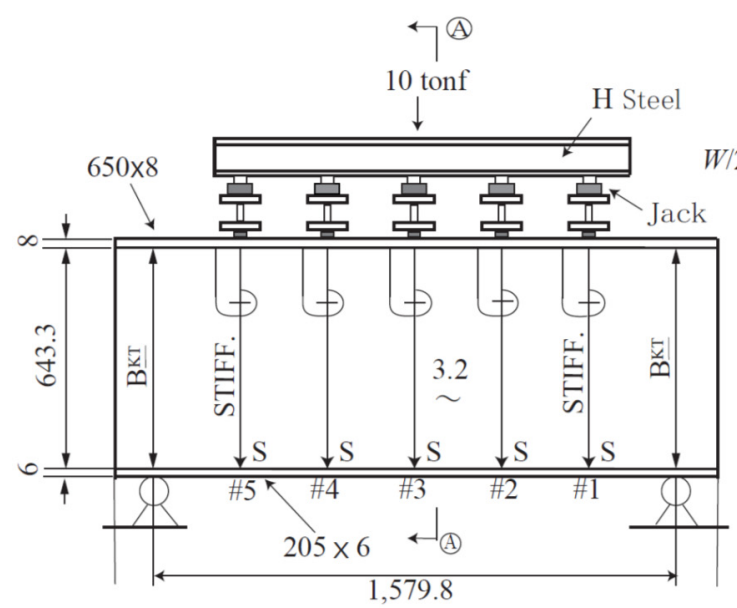

(1) Structural arrangement and loading

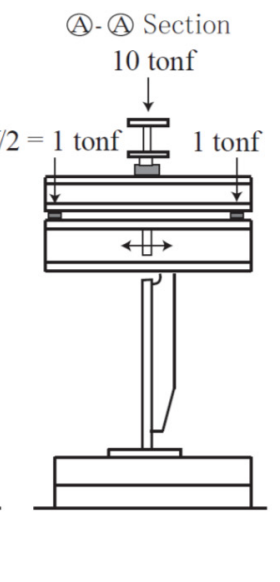

Fig. 11 Experimental set up [2]

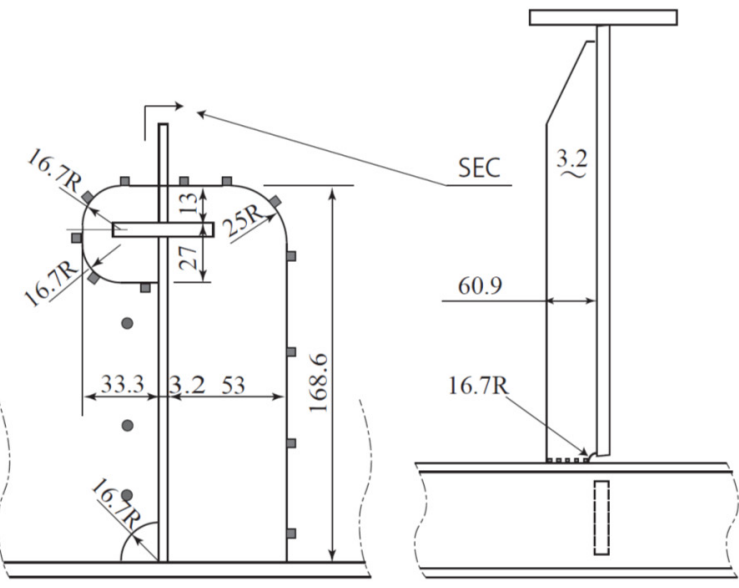

(2) Details around the slot cut-out

\subsection{Finite element analysis}

Finite element analysis is now conducted for the structures and loading as shown in Fig. 11. The 3-dimensional finite element model, loading and boundary conditions are shown in Fig. 12. Shell elements are applied all over the model. Both ends of the face plate of the primary supporting member are simply supported. Shell plate edges in way of the ends of the longitudinal stiffeners are constrained to $z$-direction to avoid torsion around $x$-axis. Four elements are arranged along the attachment of the web stiffener to the face plate of the longitudinal stiffener.

Multi-purpose finite element analysis software "zplate" is used in this analysis.

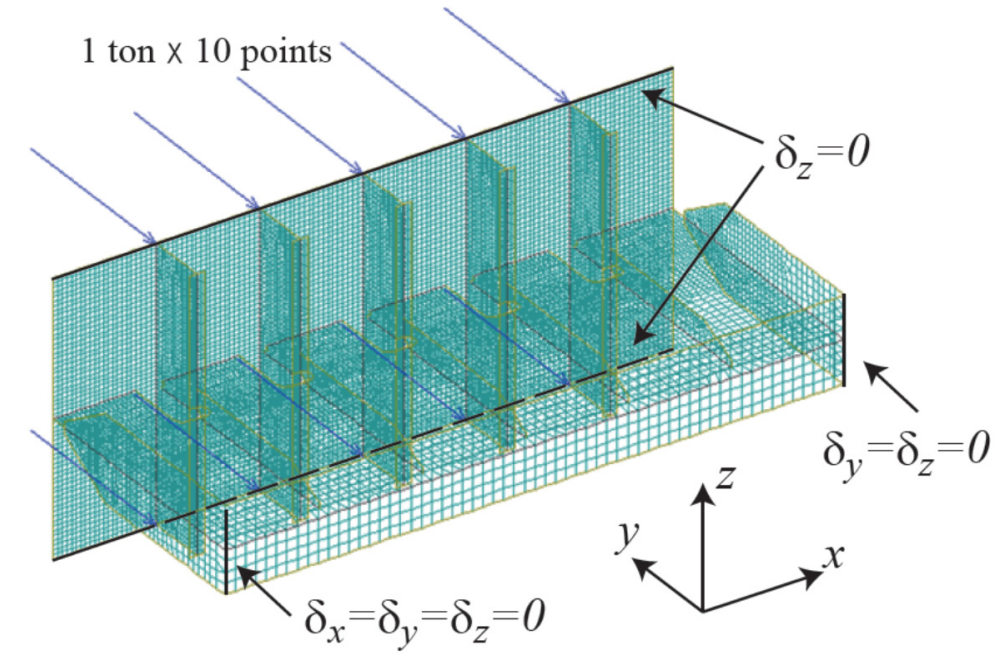

Fig. 12 Finite element model, loading and boundary conditions

\subsection{Comparison of the results among theoretical formula, finite element analysis and experiment}

Each longitudinal stiffener is numbered as shown in Fig. 13. Stiffener \#5 corresponds to $n=-2$, and stiffener \#1 corresponds to $n=2$, where $n$ is the number of longitudinal stiffeners counted from the location where the shear force on the primary supporting member is zero, as introduced in Eq.(27). When the slot cut-out is open in the left side of the longitudinal stiffeners as shown in the figure, the stress at the root of the web stiffener from the direct load from the longitudinal stiffener and the stress due to the shear force on the primary supporting member web superimpose each other when $n<0$, while they cancel each other when $n>0$.

The share of the loading supported by the web stiffener $\left(P_{f} / W\right)$ is obtained from the derived theoretical formula Eq.(32), finite element analysis and the experiment from the literature, and compared in Fig. 14. For reference, results of the formula in Reference [3] are also plotted in the graph. The parameters used in the theoretical formula are tabled in Table 2. It is noted that for the amount of $b_{1}$, half of the corner radius is deducted from the cut-out breadth. We can observe that the results of the formula agree fairly well with the results of the finite element analysis, and also they reproduce the tendency observed in the results of the experiment. On the other hand, it is found that the results of Reference [3] overestimate the load share of the web stiffener, although the inclination of the curve due to the effect of the shear force on the primary supporting member show the same tendency. 
It is confirmed that the derived formula can accurately express the complicated load transfer behavior around the slot cut-out structures by taking account of all the major structural members affecting the load path, especially thanks to the proper modeling of equivalent length of the web stiffener and the elastic spring of the primary supporting member web attached to the web stiffener for the direct force from the longitudinal stiffener.

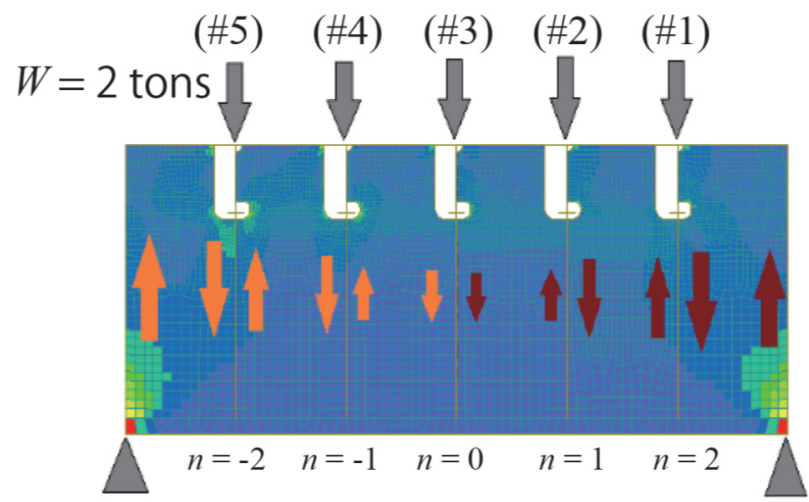

Fig. 13 Numbering of longitudinal stiffeners

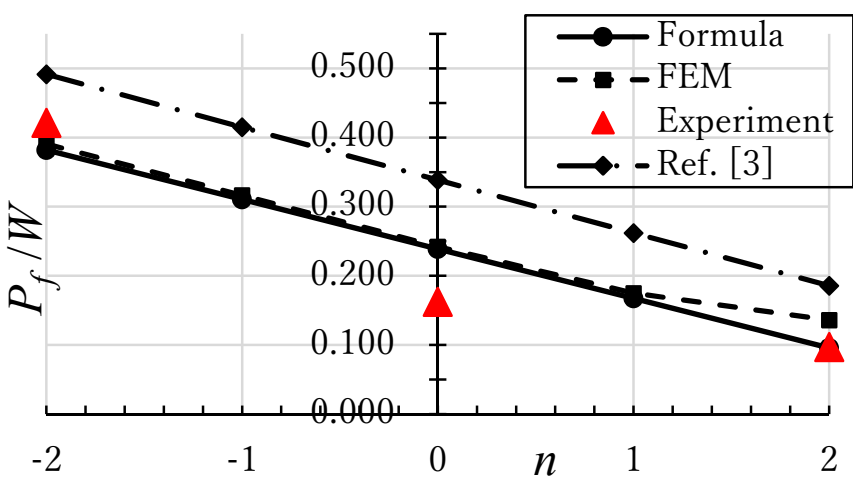

Fig. 14 Comparison of the results among theoretical formula, finite element analysis and experiment

Table 2 Parameters used in the verification study (unit: $\mathrm{mm}$ )

\begin{tabular}{|l|l|l|l|l|l|l|l|l|l|}
\hline$b_{1}$ & $b_{2}$ & $h_{1}$ & $h_{3}$ & $t_{w}$ & $b_{f}$ & $b_{f o}$ & $h_{f o}$ & $h_{s o}$ & $t_{f}$ \\
\hline 24.95 & 53 & 111.9 & 474.7 & 3.2 & 60.9 & 44.2 & 16.7 & 13 & 3.2 \\
\hline
\end{tabular}

Next, the stress distribution along the connection between the web stiffener and the face plate of the longitudinal stiffener is discussed. Fig. 15 shows the comparison among the results of the theoretical formula derived in Section 2.2, finite element analysis and experiment. Stiffeners \#1, \#3 and \#5 are selected for comparison because the experimental results are available only for these three longitudinal stiffeners. The horizontal axis designates the four equally spaced locations along the connection between the web stiffener and the longitudinal stiffener, corresponding to the center of the four elements along the connection (see Fig. 16). The results of the finite element analysis are the normal stress to the direction of the web stiffener at the center of each element. The stress distribution obtained by the experiment from the literature is shown in Fig. 17 for reference. The stresses at the location a, b, c and d in Fig. 15 are interpolated from the stresses at the five locations where the strain gauges were instrumented.

We can observe good agreement among the results of the theoretical formula, the finite element analysis and the experiment, with a slightly steeper inclination of the curve than the finite element analysis and the experiment. This slightly greater slope is considered to have been caused by the assumption that the primary supporting member is rigid as explained in Section 2.2. If the primary supporting member is assumed to be flexible, the neutral axis of the web stiffener attached to the primary supporting member changes closer to the center of the web stiffener, and the exerted bending moment by the axial force is reduced. However, observing the slight difference between the results of the formula and the finite element analysis, this deviation can be considered to be good conservatism. The peak stresses observed near the scallop by the finite element analysis and the experiment are considered to come from the stress concentration due to the effect of the scallop.

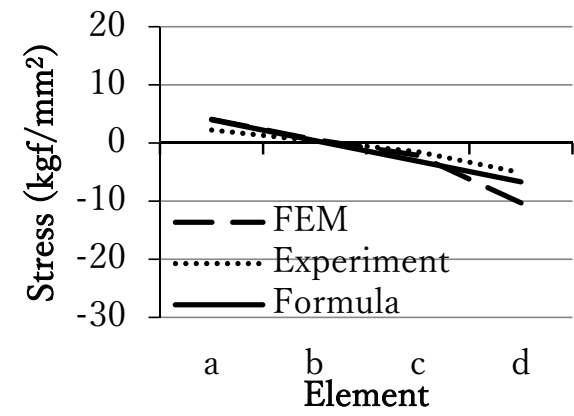

(1) Longitudinal stiffener \#1

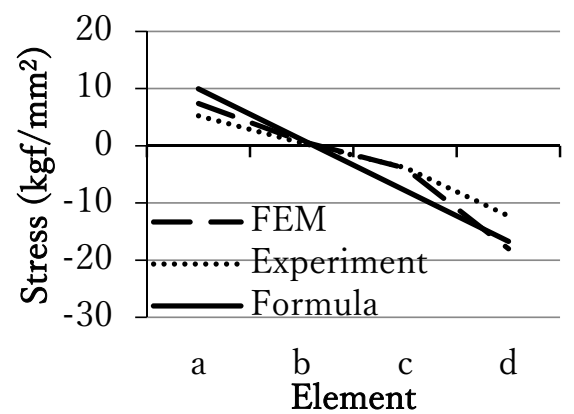

(2) Longitudinal stiffener \#3

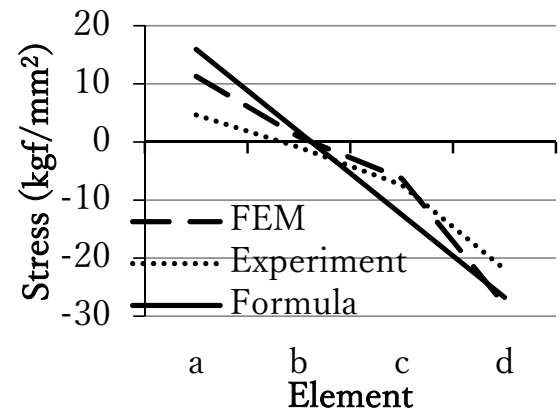

(3) Longitudinal Stiffener \#5

Fig. 15 Comparison of stress distribution along the root of the web stiffener 


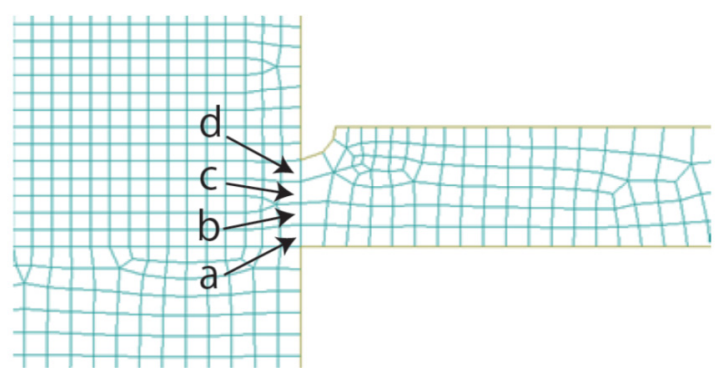

Fig. 16 Arrangement of elements along the root of the web stiffener
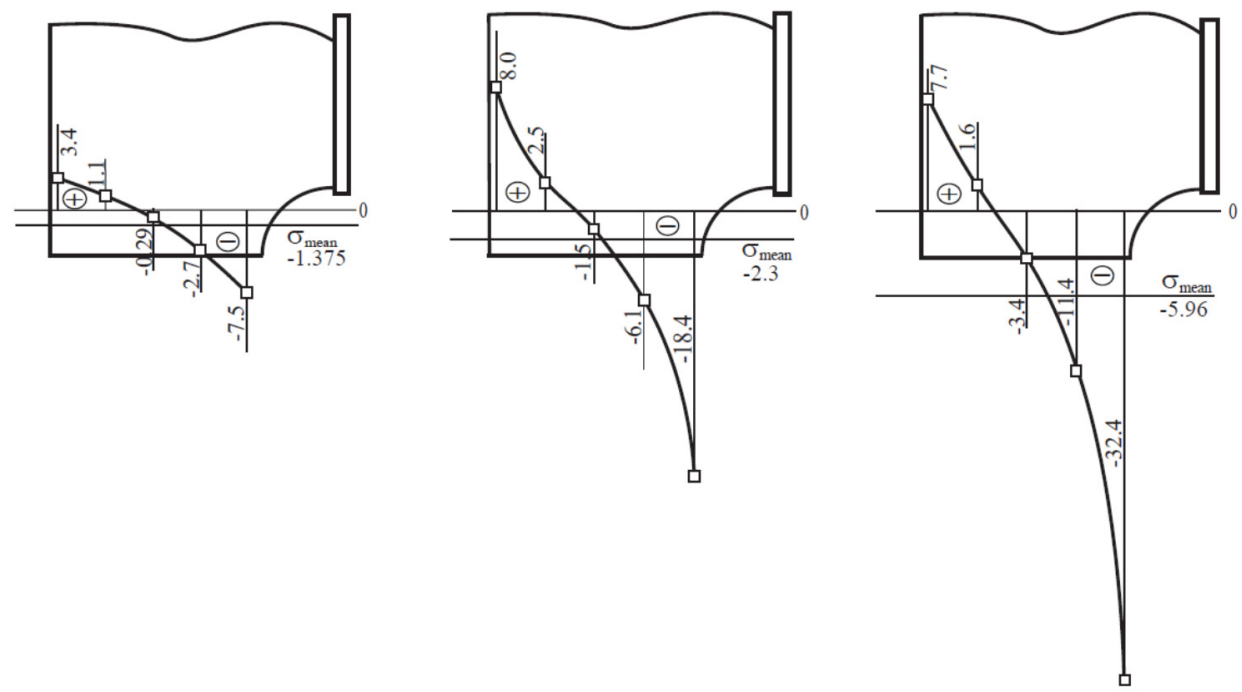
(1) Longitudinal stiffener \#1
(2) Longitudinal stiffener \#3
(3) Longitudinal Stiffener \#5

Fig. 17 Stress distribution along the root of the web stiffener from the experiment (unit: $\mathrm{kgf} / \mathrm{mm}^{2}$ ) [2]

\section{PARAMETRIC STUDIES}

In this chapter, the authors deal with a typical slot cut-out structure of the parameters as shown in Table 3, for which share of the loads among the web stiffener and the primary supporting member web is calculated. In the Case 1 , a collar plate is not fitted, and one side of the slot cut-out is open. Therefore, $h_{2}$ and $t_{c}$ in Table 3 are set to be zero in this case.

Table 3 Parameters of a typical slot cut-out structure (unit : mm)

\begin{tabular}{|l|l|c|c|c|c|c|c|c|c|c|c|c|c|}
\hline & & $b_{1}$ & $b_{2}$ & $h_{1}$ & $h_{2}$ & $h_{3}$ & $t_{w}$ & $t_{c}$ & $b_{f}$ & $b_{f o}$ & $h_{f o}$ & $h_{s o}$ & $t_{f}$ \\
\hline Case 1 & Base & 92 & 139 & 284 & 0 & 915 & 13 & 0 & 250 & 200 & 50 & 35 & 16 \\
\hline Case 2 & 350x22 web stiffener & 92 & 139 & 284 & 0 & 915 & 13 & 0 & $\mathbf{3 5 0}$ & $\mathbf{3 0 0}$ & 50 & 35 & $\mathbf{2 2}$ \\
\hline Case 3 & Collar plate added & 92 & 139 & 284 & $\mathbf{1 8 5}$ & 915 & 13 & $\mathbf{1 3}$ & 250 & 200 & 50 & 35 & 16 \\
\hline Case 4 & Slit cut-out & 92 & $\mathbf{9 2}$ & 284 & $\mathbf{2 8 4}$ & 915 & 13 & $\mathbf{1 3}$ & 250 & 200 & 50 & 35 & 16 \\
\hline
\end{tabular}

Fig. 18 (1) shows the results of the calculation of the share of loading of each member, taking $n$ for the horizontal axis. It is observed that when $n=-5$, the amount of loading transferred to the web stiffener is already greater than the amount of total loading $W$ from the longitudinal stiffener $(105.8 \%$ of $W)$, while when shearing force is not acting $(n$ $=0$ ), the amount of loading transferred to the web stiffener is $39.4 \%$ of the total loading. Such a large loading to the web stiffener may cause structural failures if the scantling of the web stiffener is not properly designed to withstand this high share of loading. Then, we consider some different methods to reinforce this structure to reduce the average stress in way of the root of the web stiffener.

To reduce the stress of the web stiffener, the most direct way is to increase the scantling of the web stiffener. In the Case 2, the web stiffener scantling is increased to $b_{f} \times t_{f}=350 \times 22$ instead of $250 \times 16$, where $b_{f}$ includes width of the scallop. The length of the connection between the web stiffener and the longitudinal stiffener $b_{f o}$ is changed to $300 \mathrm{~mm}$ considering the same scallop size of $50 \mathrm{~mm}$. The figures changed from Case 1 is shown in bold font in Table 3. Fig. 18 (2) shows the results of loading share carried by each structural component. The sectional area at the connection between the web stiffener and the longitudinal stiffener is increased from $200 \times 16=3200 \mathrm{~mm}$ to $300 \times$ $22=6600 \mathrm{~mm}$, i.e. 2.06 times the original value. We can observe that due to the increase of the spring constant of the web stiffener, the share of loading of the web stiffener increased prominently from $105.8 \%$ in Fig. 18 (1) to 
$128.0 \%$ in Fig. 18 (2) in case of $n=-5$. As a result, exerted stress reduces to $(1.280 / 6600) /(1.058 / 3200)=0.587$, i.e. about $58.7 \%$ of the original stress, although the scantling is increased to more than twice the original structure.

Next, in Case 3, reinforcement by the attachment of a collar plate is considered. A collar plate of $185 \mathrm{~mm}$ height $\left(h_{2}\right)$ and $13 \mathrm{~mm}$ thickness $\left(t_{c}\right)$ is fitted. The resulting share of loading is shown in Fig. 18 (3). It is observed that the share of loading by the web stiffener drastically reduced to $43.6 \%$ of the total loading when $n=-5$. This is about $43.6 \%$ / $105.8 \%=41.2 \%$ of the original stress. We can observe from Fig. 18 (3) that the inclination of the curve of $P_{f} / W$ decreased prominently compared with Fig. 18 (1) and (2). The reason can be attributed to the effect of the collar plate, which balances the shear force from both the sides of the longitudinal stiffener, and therefore, reduces the effect of shear force of the primary supporting member to the stress exerted on the web stiffener.

With regard to this balancing effect, completely symmetric slot cut-out structure can be achieved by applying the socalled slit cut-out, where a narrow slit to pass a longitudinal stiffener web and an open hole to pass a longitudinal stiffener face plate are cut on the primary supporting member web. The longitudinal stiffener web plate is welded to primary supporting member web plate on both the sides of the plate. This symmetric condition can be simulated by setting $b_{2}=b_{1}=92 \mathrm{~mm}, h_{2}=h_{1}=284 \mathrm{~mm}$, and $t_{c}=t_{w}=13 \mathrm{~mm}$ as shown as Case 4 in Table 3 . The results are shown in Fig. 18 (4). It is observed that the share of loading by the web stiffener becomes completely constant irrespective of the magnitude of the shear force on the primary supporting member. The share of loading by the web stiffener further reduced to $22.1 \%$ of the total loading irrespective of the value of $n$, corresponding to $22.1 \% / 105.8 \%=20.9 \%$ of the original stress. Increased spring constant of the shear connection between the primary supporting member web and the longitudinal stiffener web is considered to have contributed to the reduction of the share of loading supported by the web stiffener.

Last but not least, we can consider another countermeasure to arrange the slot cut-out to the opposite direction. This condition corresponds to the case of $n=5$ in Fig. 18 (1). In this case, the share of the loading by the web stiffener is $-26.9 \%$ of the total loading, as a result of cancellation of stresses between the effect of the loading from the longitudinal stiffener and the effect of the shear force on the primary supporting member. Minus sign means that the effect of the shear force on the primary supporting member was greater than the effect of the loading from the longitudinal stiffener. This share of loading corresponds to $-26.9 \% / 105.8 \%=-25.4 \%$ of the original stress.

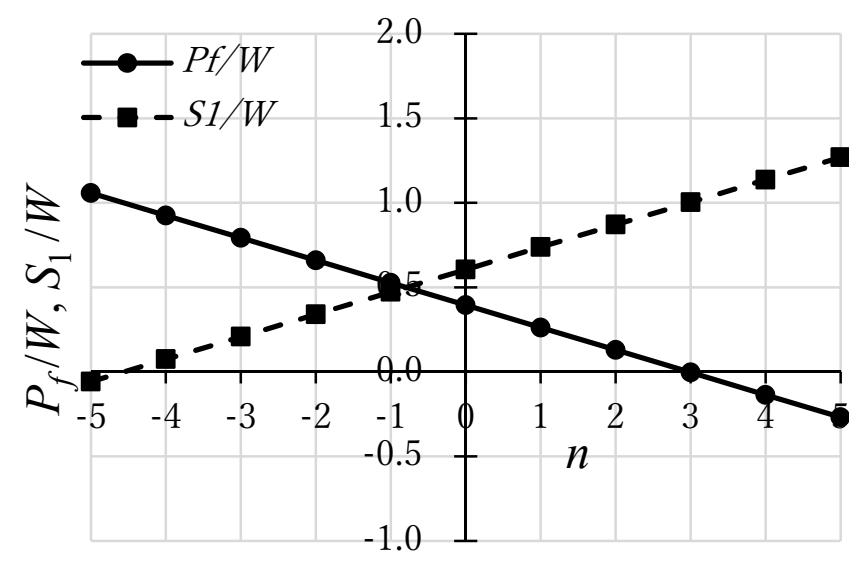

(1) Case 1: one side of the slot cut-out is open

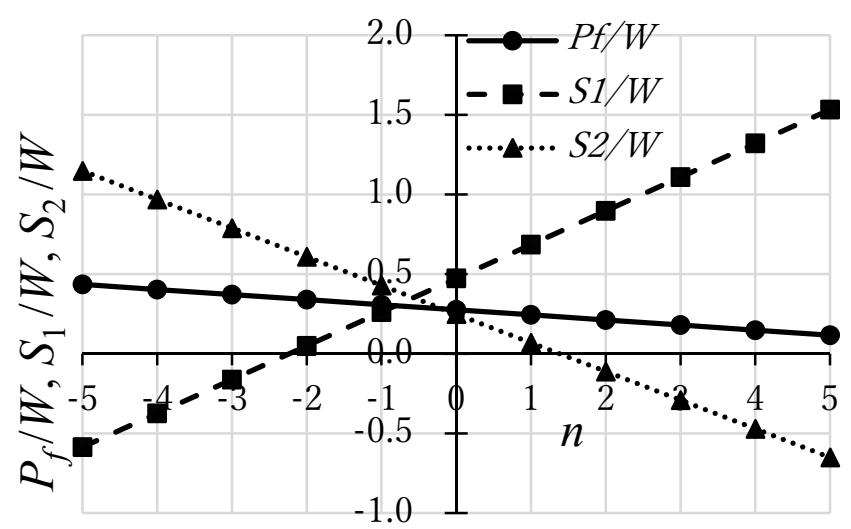

(3) Case 3: A collar plate is fitted

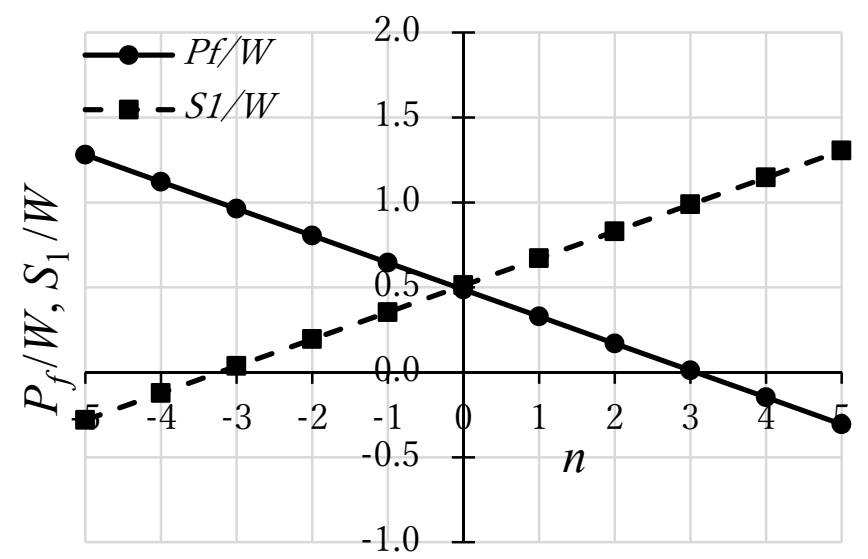

(2) Case 2: Web stiffener reinforced to $350 \mathrm{~mm} \times 22 \mathrm{~mm}$

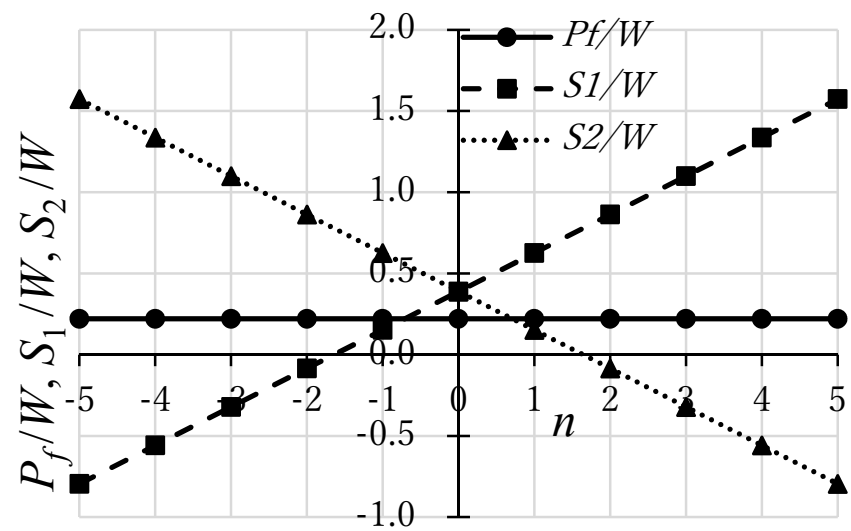

(4) Case 4: Slit cut-out structure

Fig. 18 Share of loading among web stiffer, primary supporting member web and collar plate for various types of reinforcement 
Installation of a backing bracket is a costly alternative, requiring additional sub-assembly process of the reverse side of the primary supporting member web. However, it is considered to be a very effective countermeasure to mitigate the stress at the root of the web stiffener, because not only it reduces the nominal stress thanks to the increased sectional area, but also it balances the forces exerted on the web stiffener, and therefore, the local increase in the stress due to the bending of the web stiffener as illustrated in Fig. 9 is substantially alleviated. The formulation of the effect of the back bracket is derived in Reference [3]. In this paper, the effect of the back bracket is not included and is left to be one of the important tasks to be implemented in the future.

Reduction of stresses in case of the countermeasures stated above is summarized in Table 4. We can conclude that:

1. Most direct countermeasure, i.e., to increase the scantling of the web stiffener, works, but because the share of the loading born by the web stiffener also increases due to the increase in the spring constant of the web stiffener, the stress reduction ratio is less than the scantling increase ratio.

2. Fitting of a collar plate is more effective way to mitigate the effect of the shear force on the primary supporting member, because the shear forces on both the sides of the slot cut-out balances to some extent. Application of completely symmetric slit cut-out structure is further effective.

3. It may be another effective countermeasure to direct the slot cut-out to the direction where the stress due to the loading from the longitudinal stiffener and the stress due to the shear force on the primary supporting member cancel each other. This is the most cost effective way, needing no additional members nor scantling increase. However, this countermeasure may not be always possible depending on the construction processes and facilities.

Table 4 Summary of stress reduction in case of various types of reinforcement

\begin{tabular}{|l|c|c|}
\hline & $\begin{array}{c}\text { Share supported by web stiffener } \\
P_{f} / W(\%)\end{array}$ & \begin{tabular}{c} 
Stress ratio to the base case (\%) \\
\hline Case 1: Base case
\end{tabular} $105.8 \%$ \\
\hline Case 2: Web stiffener reinforced & $128.0 \%$ & $(100 \%)$ \\
\hline Case 3: Collar plate fitted & $43.6 \%$ & $58.7 \%$ \\
\hline Case 4: Slit cut-out structure & $22.1 \%$ & $41.2 \%$ \\
\hline Slot cut-out direction changed & $-26.9 \%$ & $20.9 \%$ \\
\hline
\end{tabular}

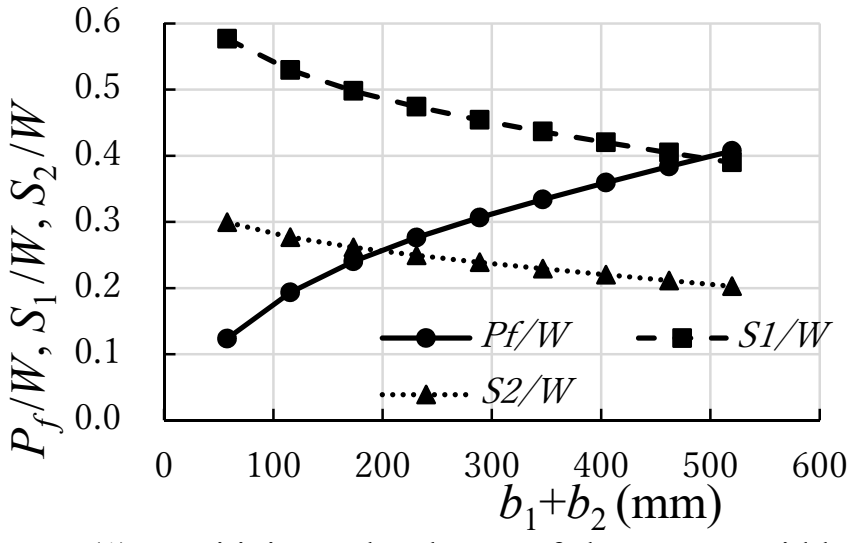

(1) Sensitivity to the change of slot cut-out width

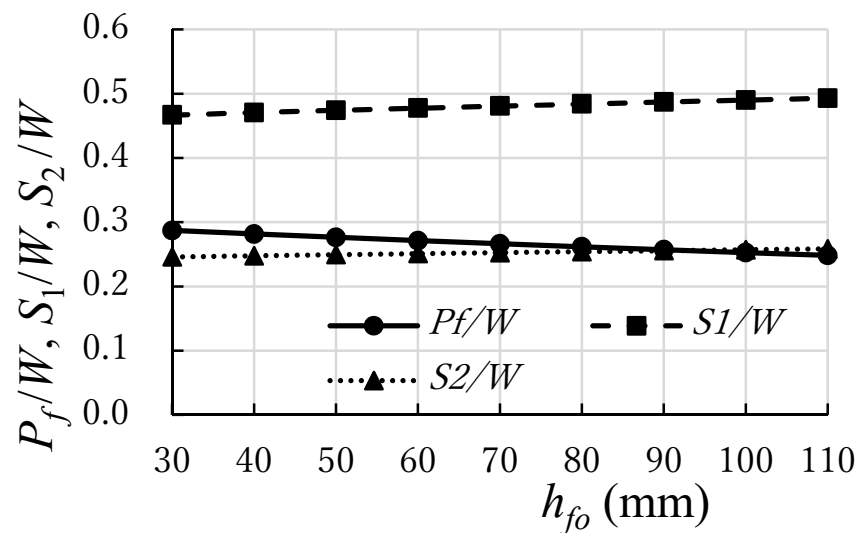

(2) Sensitivity to the change of scallop height

Fig. 19 Share of loading born by each member according to the change of various parameters

Next, sensitivity of the stress at the root of the web stiffener to the change of some parameters is studied using the derived theoretical formula. Firstly, the slot cut-out width $b_{1}$ and $b_{2}$ are changed proportionately, and the resulting share of loading supported by each member is shown in Fig. 19 (1), taking $b_{1}+b_{2}$ for the horizontal axis. Parameters as shown as Case 3 in Table 3 with a collar plate is adopted for this study. In this study, $n$ is set to be zero, i.e., the shear force on the primary supporting member is not working in this case. From Fig. 19 (1), we can find that when the width of the slot cut-out becomes greater, the share of loading born by the web stiffener increases quite significantly, due to the reduction of the shear spring constant in way of the primary supporting member web and the collar plate attached to the longitudinal stiffener web plate. Therefore, we should avoid too wide face plate of the longitudinal stiffener especially in case of L-shaped stiffener, which may result in wider width of the slot cut-out. Next analysis is to the change of the scallop height $\left(h_{f o}\right)$ at the heel of the web stiffener. Slot cut-out height above the face plate of the longitudinal stiffener $\left(h_{s o}\right)$ is changed in accordance with the scallop height. Fig. 19 (2) shows the share of loading supported by each member, taking the scallop height for the horizontal axis. As the scallop becomes taller, the share of loading born by the web stiffener decreases, because of the reduction of the spring constant at the root of the web stiffener. However, this reduction is observed to be quite mild. Because taller scallop height causes 
weaker buckling strength around the root of the web stiffener, application of taller scallop should be carefully reviewed for compressive stresses.

\section{CRITICAL REVIEW ON RULE FORMULAE}

In 2014, IACS (International Association of Classification Societies) published "Common structural rules for bulk carriers and oil tankers (CSR)" [10], which is commonly applicable to bulk carriers with length greater than or equal to $90 \mathrm{~m}$ and oil tankers with length greater than or equal to $150 \mathrm{~m}$. In Part I Chapter 3 Section 6.5 of the rules, scantling requirement is stipulated with regard to the intersection of stiffeners and primary supporting members. The rule formula stipulating the load carried by the web stiffener is shown in Eq.(84) using the nomenclature of this paper.

$$
P_{f}=W\left(1-\alpha_{a}-\frac{h_{1} t_{w}+h_{2} t_{c}}{4 f_{c} b_{f o} t_{f}+h_{1} t_{w}+h_{2} t_{c}}\right)
$$

where $\alpha_{a}$ is the aspect ratio of the skin panel, i.e., longitudinal stiffener spacing divided by transverse frame spacing, and

$$
\begin{aligned}
& f_{c}=1.85 \\
& f_{c}=1.85-0.000441\left(b_{f o} t_{f}-1400\right) \text { for } 1400<b_{f o} t_{f} \leq 3100\left[\mathrm{~mm}^{2}\right] \\
& f_{c}=1.1-0.00013\left(b_{f o} t_{f}-3100\right) \quad \text { for } 3100<b_{f o} t_{f} \leq 5800\left[\mathrm{~mm}^{2}\right] \\
& f_{c}=0.75 \quad \text { for } b_{f o} t_{f}>5800\left[\mathrm{~mm}^{2}\right]
\end{aligned}
$$

As we can observe, the IACS rule formula (CSR) takes account of only the load component directly transferred from the longitudinal stiffener, and do not consider the effect of the shear force on the primary supporting member.

Using the example shown in Chapter 3 of this paper, IACS rule formula is applied and the resulting load share born by the web stiffener is plotted in Fig. 20 with a dot-and-dash line. The panel aspect ratio $\alpha_{a}$ is assumed to be 0.34 in this case, and $f_{c}$ is assumed to be 1.85 because $b_{f o} t_{f}=141.44 \mathrm{~mm}^{2}$ is less than $1400 \mathrm{~mm}^{2}$ even if further multiplied by 9 to account for the actual scale which is 3 times larger than the middle scale model. Because the effect of the shear force is neglected, the load share does not change according to $n$.

We can observe that when the shear force is not acting $(n=0)$, the calculated load share by IACS rule formula is sufficiently overestimated, but when $n=-2$, it is almost equivalent to the value estimated by the proposed formula in this paper, finite element analysis and the experiment. This means that when $n$ is less than or equal to -3 , which is very probable in actual structures, IACS rule formula will underestimate the resulting load share by the web stiffener. This may result in insufficient strength in way of the root of the web stiffener around the high shear force region of primary supporting members.

On the contrary, when the slot cut-out is directed so that the effect of the shear force on the primary supporting member cancels the effect of the load from the longitudinal stiffener, IACS rule formula is considered to be too conservative.

Therefore, it is recommendable to use the rational theoretical formula of load share of each member as proposed in this paper to realize safe and rational slot cut-out structures.

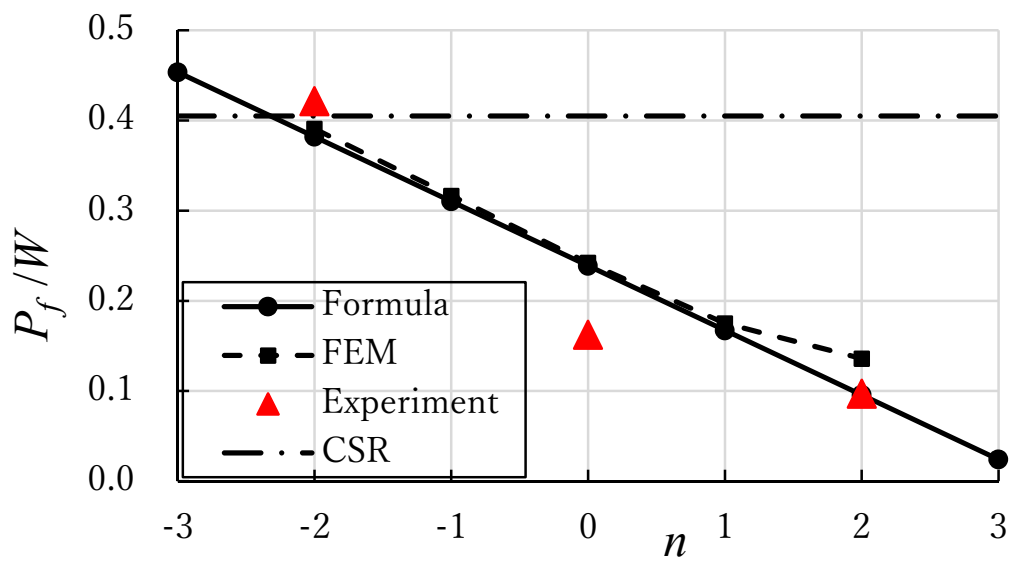

Fig. 20 Comparison of load share by each member between IACS CSR and the proposed formula

Next, IACS rule formula is applied to the examples Case $1 \sim$ Case 4 , studied in Chapter 4 in this paper. Comparison of the load share carried by the web stiffener between the proposed formula and IACS rule formula is shown in Fig. 
21. In this case, the panel aspect ratio $\alpha_{a}$ is assumed to be 0.25 , and $f_{c}$ is calculated to be 1.087 according to Eq.(85). We can find that when the shear force on the primary supporting member web is not working $(n=0)$, IACS rule formula always gives greater estimate than the proposed formula. On the other hand, under the action of the shear force on the primary supporting member, the proposed formula gives greater estimate already when $n=-1$ or -2 if the slot is open on one side (Case 1 and Case 2), meaning that the effect of the shear force is significant and can be underestimated using IACS rule formula in this case, too.

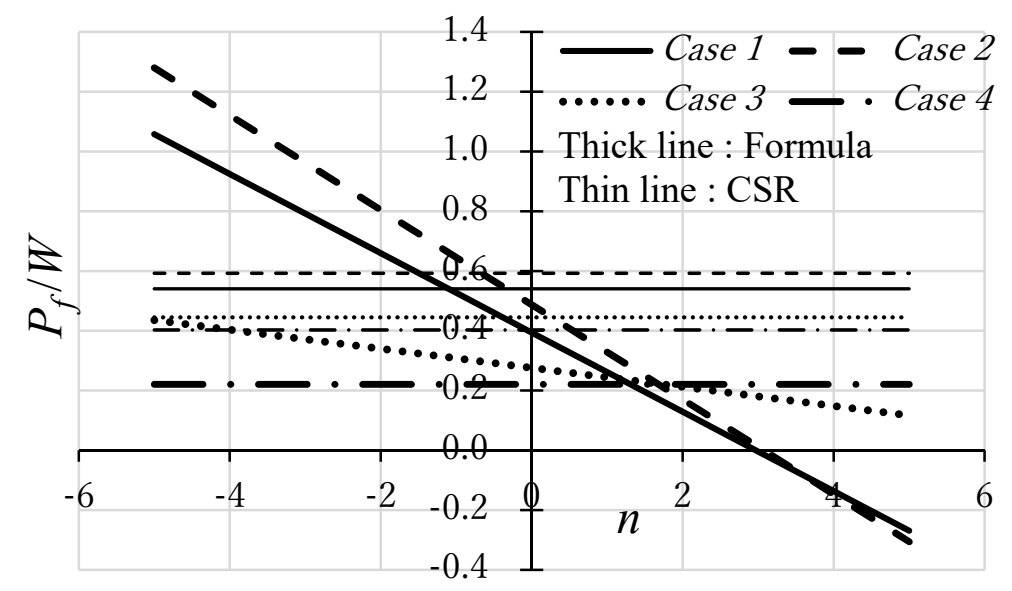

Fig. 21 Comparison of load share carried by web stiffener between IACS CSR and the proposed formula for Cases 1 4

\section{CONCLUSIONS}

In this paper, the authors established a theoretical formula to represent the stresses at the root of the web stiffener due to the load from both the longitudinal stiffener and the shear force on the primary supporting member. The derived formula was verified through comparison with the results of finite element analysis and past experiments. Then, various parametric studies and critical review on the rules are conducted using the derived formula. As a result, the following concluding remarks can be made.

1. Theoretical formulae in some of the past literatures did not take account of the elastic spring of the primary member web plate above the slot cut-out. Therefore, they overestimated the load share born by the web stiffener, and empirical correction coefficient was introduced using damage and no-damage experiences or calibration based on finite element analysis. On the other hand, a literature [3] took account of all the structural components. However, the derived formulae were too cumbersome for practical application due to its exhaustiveness, and also they were not verified through finite element analyses nor experiments. In this study, consistent theoretical formula is developed taking account of all the structural components affecting the load share of each member. Simple and theoretical derivation of the formulae is pursued as far as possible to facilitate practical application. As a result of comparison with finite element analysis and past experiments, good accuracy of the proposed formula was verified.

2. When one side of the slot cut-out is open and directed so that the effect of the load from the longitudinal stiffener and the load from the shear force on the primary supporting member superimpose each other, the stresses at the root of the web stiffener may become too large. Among various countermeasure, most direct countermeasure may be to increase the scantling of the web stiffener, but because the share of the loading born by the web stiffener also increases due to the increase in the spring constant of the web stiffener, the stress reduction ratio is less than the scantling increase ratio. Fitting of a collar plate is more effective way to mitigate the effect of the shear force on the primary supporting member, because the shear forces on both the sides of the slot cut-out balances to some extent. Application of completely symmetric slit cut-out structure is further effective. It is the most cost effective way to direct the slot cut-out to the direction where the stress due to the loading from the longitudinal stiffener and the stress due to the shear force on the primary supporting member cancel each other.

3. IACS CSR formulae of the slot cut-out structures take account of only the direct load from the longitudinal stiffeners, and do not consider the effect of the shear force on the primary supporting member. Therefore, it is probable that the rules give insufficient strength when the effect of the shear force on the primary supporting member superimposes the effect of the load from the longitudinal stiffener. On the other hand, the rules may be too conservative when they cancel each other. It is recommendable to use the rational theoretical formula of load share of each member as proposed in this paper to realize safe and rational slot cut-out structures. 
The authors would like to thank Mr. Masahiro Fujiwara for his valuable advices with regard to the derivation of theoretical formulae on slot cut-out structures. The finite element analysis in this paper was mainly carried out by Mr. Shogo Kubota as his studies for the PED program portfolio in Yokohama National University. His contribution to this study is much appreciated.

\section{REFERENCES}

[1] Okumoto Y, Takeda Y, Mano M, Okada T. Design of ship hull structures - A practical guide for engineers. Springer; 2009.

[2] Ochi Y, Nagano T, Yamaguchi K, Kimura H, Kashima H. Strength around slot for penetration of longitudinals. IHI Engineering Review 1971; 11:6; 463-474, in Japanese.

[3] Research Committee of the West-Japan Society of Naval Architects. Surveillance study for ship structural damages (2) - Damages around slot structure in tanks -. Research report No.13 of the West-Japan Society of Naval Architects, 1971, in Japanese.

[4] Matoba M, Imamura N, Hasegawa Y, Yonesato T, Inoue H. Fatigue strength of slotted parts of ship transverse web frame. Journal of the Society of Naval Architects of Japan 1974; 136; 371-383, in Japanese.

[5] The $120^{\text {th }}$ Research Committee of the Shipbuilding Research Association of Japan (SR120). On the prevention of crack around cut-outs in bottom transverses of large ships. Report No.76, The Shipbuilding Research Association of Japan, 1974, in Japanese.

[6] Nakajima Y, Iino N, Neki I, Ushirokawa O, Maeda M, Sasajima H. Fatigue strength design of ship structure. IHI Engineering Review 1993; 26:2.

[7] Watanabe E, Inoue S, Hashimoto K, Sato K, Sueoka H. Proposal of simplified fatigue design method for side longitudinals. Journal of the Society of Naval Architects of Japan 1995; 177; 391-398

[8] Seki N, Okada T, Okuzuka G. Practical considerations on the third generation fatigue damage of slot cut-out structure. $11^{\text {th }}$ International Symposium on Practical Design of Ships and Other Floating Structures (PRADS 2010), Rio de Janeiro; 2010. pp.1270-1279.

[9] Okada T. Double hull tanker structures - Some practical considerations about CSR applications. Tanker Structure Cooperative Forum 2010 Shipbuilders Meeting; 2010.

[10] IACS (International Association of Classification Societies). Common structural rules for bulk carriers and oil tankers. 2014.

[11] Kaase G.O.R. Fatigue strength verification of stiffener-frame connections - DNV Class notation PLUS. $11^{\text {th }}$ International Symposium on Practical Design of Ships and Other Floating Structures (PRADS 2010), Rio de Janeiro; 2010. pp.1259-1269.

[12] Ma K-T, Srinivasan S, Zhang H, Healy B, Peng H. Developing design criteria for connections around cutout (slot) openings. SNAME Transactions 2000; 108; 227-248.

\section{APPENDIX A. EQUIVALENT LENGTH OF THE WEB STIFFENER}

Spring constant of the web stiffener is very important to accurately estimate the share of loading supported by each member. However, because the load transferred into the web stiffener is gradually transferred into the primary supporting member web through the connection between the web stiffener and the primary supporting member web, all the length of the web stiffener does not work as a spring. Therefore, it is necessary to estimate equivalent length of the web stiffener, working as a spring. Reference [3] obtained this shear stress distribution along the attachment between the web stiffener and the primary member web based on the results of parametric finite element analyses. Here, in this paper, the equivalent length of the web stiffener is obtained using the beam theory as developed in Section 2.2.

Assuming the arrangement and coordinate as shown in Fig. 8, the elongation of the web stiffener, $u_{f}$, at the base $(x=$ 0 ) is obtained as follows from Eq.(40) considering that the slope of the web stiffener is zero.

$$
u_{f}=\frac{f_{w}}{E k_{w}}
$$

Substituting Eq.(60) into Eq.(A.1), putting $x=\xi=0$, 


$$
\begin{aligned}
u_{f} & =-\frac{P_{f}}{E k_{w}} \frac{\lambda}{h_{f}} \frac{1}{\tanh \lambda}=-\frac{P_{f}}{E k_{w}} \frac{1}{\tanh \lambda} \frac{1}{h_{f}} 2 \sqrt{k_{w}\left(\frac{b_{f}}{t_{f}}\right)}\left(\frac{h_{f}}{b_{f}}\right) \\
& =-\frac{P_{f}}{E b_{f}} \frac{2}{\tanh \lambda} \sqrt{\frac{1}{k_{w}}\left(\frac{b_{f}}{t_{f}}\right)}
\end{aligned}
$$

Alternatively, using the equivalent length $h_{e}$ of the web stiffener,

$$
u_{f}=-\frac{P_{f} h_{e}}{E b_{f} t_{f}}
$$

From comparison between Eq.(A.2) and Eq.(A.3), the equivalent length is obtained as follows.

$$
\frac{h_{e}}{t_{f}}=\frac{2}{\tanh \lambda} \sqrt{\frac{1}{k_{w}}\left(\frac{b_{f}}{t_{f}}\right)}
$$

Next, let us calculate the magnitude of the shear spring at the connection, $k_{w}$. Assuming the double-sided welding bead and the coordinate as shown in Fig. A.1, shear spring for the displacement perpendicular to the paper surface can be calculated as the integration of the shear spring of the infinitesimal strips whose width is $d x$ and the length is $b(x)$, and is expressed as follows.

$$
k_{w}=\frac{2 G}{E} \int_{t_{1}}^{t} \frac{d x}{b(x)}=\frac{2 G}{E} \int_{t_{1}}^{t} \frac{d x}{2 x}=\frac{G}{E}\left(\ln t-\ln t_{1}\right)=\frac{G}{E} \ln \frac{t}{t_{1}}
$$

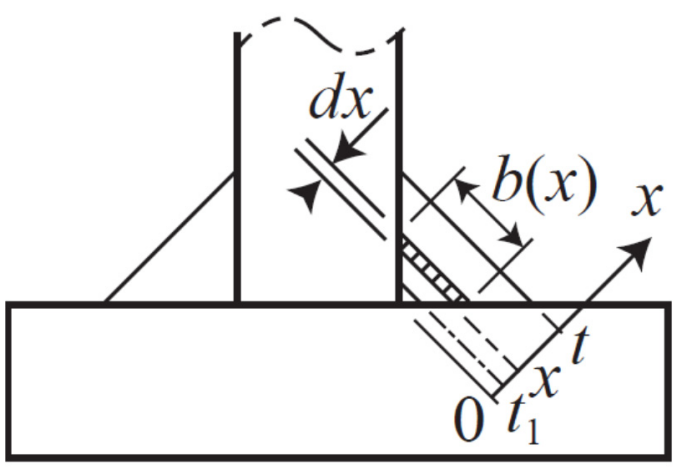

Fig. A.1 Welding bead model for shear spring calculation

When weld leg length is $5 \mathrm{~mm}$ with $1 \mathrm{~mm}$ root gap, substituting $t / t_{1}=5$ into Eq.(A.5),

$$
k_{w}=\frac{G}{E} \ln 5=\frac{\ln 5}{2(1+v)}=0.6190 \approx 0.6
$$

is obtained.

Next, let us estimate $\lambda$ under typical web stiffener configuration. $\lambda$ is defined as Eq.(49). Then assuming that typical configuration of web stiffener follows the relationship of

$$
\frac{b_{f}}{t_{f}}=18, \quad \frac{b_{f}}{h_{f}}=0.08
$$

then, $\lambda$ can be estimated to be about

$$
\begin{aligned}
& \lambda=2 \sqrt{0.6 \times 18} \times \frac{1}{0.08}=82.2 \\
& \therefore \tanh \lambda \approx 1
\end{aligned}
$$

Substituting these $k_{w}$ and $\lambda$ into Eq.(A.4), the equivalent length of web stiffener is finally obtained as follows.

$$
\frac{h_{e}}{t_{f}}=\frac{2}{\tanh \lambda} \sqrt{\frac{1}{k_{w}}\left(\frac{b_{f}}{t_{f}}\right)}=2 \sqrt{\frac{5}{3}\left(\frac{b_{f}}{t_{f}}\right)}
$$




\section{APPENDIX B. DERIVATION OF DISPLACEMENT $\delta_{3} W$}

As explained in Section 2.1, shear deflection of the primary supporting member web above the slot cut-out is different depending on whether the loading comes from the longitudinal stiffener through the web stiffener or the loading comes from the shear force on the primary supporting member. The reason is that the loading from the longitudinal stiffener is quite local, and transferred to the primary supporting member web within a relatively small zone close to the slot cut-out, while the loading from the shear force acts along the total length of the web stiffener.

In this APPENDIX, we derive the deflection of the primary supporting member web above the slot cut-out, $\delta_{3 W}$, due to only the loading from the longitudinal stiffener with no shear force on the primary supporting member.

Without the action of the shear force, the model of connection between each member and load transfer can be simplified as shown in Fig. B.2, compared with Fig. 5.
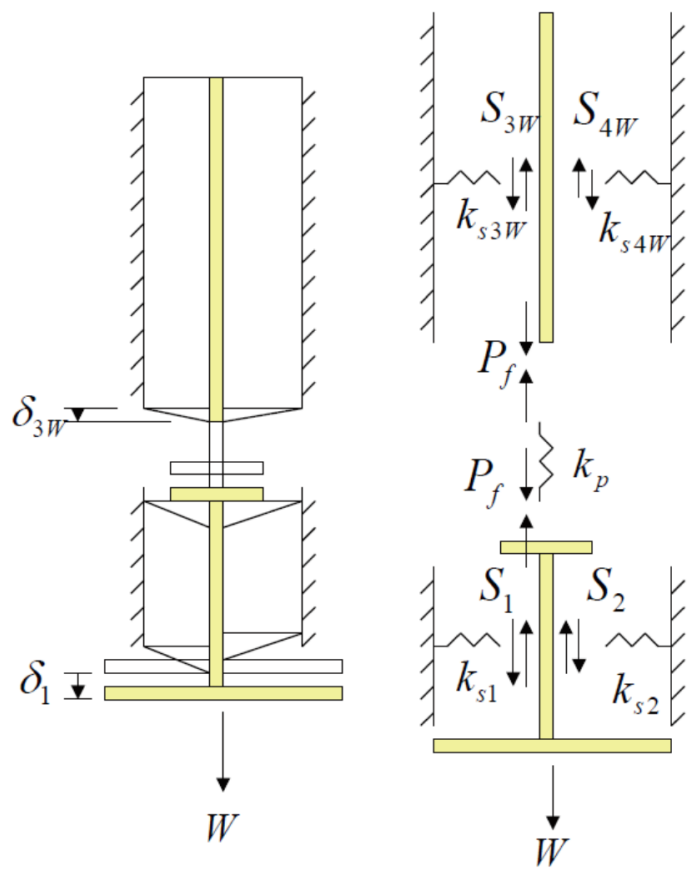

Fig. B.1 Model of connections between each member and load transfer in case of no shear force

In this case, from the equilibrium of forces at the longitudinal stiffener and the web stiffener,

$$
\begin{aligned}
& P_{f}+S_{1}+S_{2}=W \\
& S_{3 W}+S_{4 W}=P_{f}
\end{aligned}
$$

The forces acting on the web stiffener, the primary supporting member webs and the collar plate are expressed as follows.

$$
\begin{aligned}
& P_{f}=k_{p}\left(\delta_{1}-\delta_{3 W}\right) \\
& S_{1}=\alpha_{1} k_{p} \delta_{1} \\
& S_{2}=\alpha_{2} k_{p} \delta_{1} \\
& S_{3 W}=\alpha_{3 W} k_{p} \delta_{3 W} \\
& S_{4 W}=\alpha_{4 W} k_{p} \delta_{3 W}
\end{aligned}
$$

Putting Eqs.(B.3), (B.4) and (B.5) into Eq.(B.1),

$$
k_{p}\left(\delta_{1}-\delta_{3 W}\right)+\alpha_{1} k_{p} \delta_{1}+\alpha_{2} k_{p} \delta_{1}=W \quad \text { or } \quad\left(1+\alpha_{1}+\alpha_{2}\right) \delta_{1}-\delta_{3 W}=\frac{W}{k_{p}}
$$

Putting Eqs.(B.3), (B.6) and (B.7) into Eq.(B.2),

$$
-k_{p}\left(\delta_{1}-\delta_{3 W}\right)+\alpha_{3 W} k_{p} \delta_{3 W}+\alpha_{4 W} k_{p} \delta_{3 W}=0 \quad \text { or } \quad-\delta_{1}+\left(1+\alpha_{3 W}+\alpha_{4 W}\right) \delta_{3 W}=0
$$


Solving these simultaneous equation (B.8) and (B.9), $\delta_{3 W}$ is finally obtained as

$$
\delta_{3 W}=\frac{1}{\left(1+\alpha_{1}+\alpha_{2}\right)\left(1+\alpha_{3 W}+\alpha_{4 W}\right)-1} \cdot \frac{W}{k_{p}}
$$

